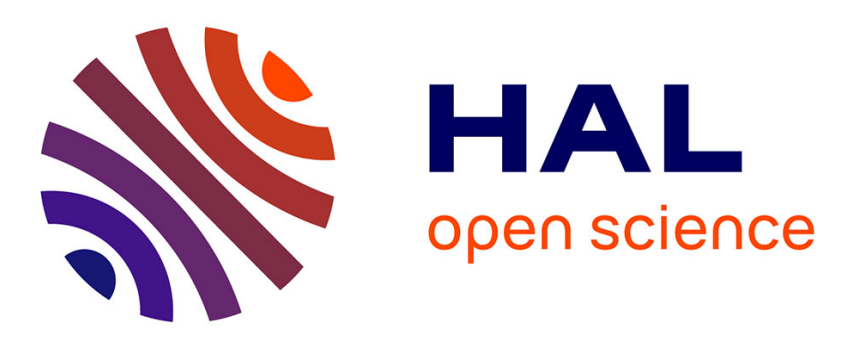

\title{
On entropy weak solutions of Hughes' model for pedestrian motion
}

Nader El-Khatib, Paola Goatin, Massimiliano D. Rosini

\section{To cite this version:}

Nader El-Khatib, Paola Goatin, Massimiliano D. Rosini. On entropy weak solutions of Hughes' model for pedestrian motion. Zeitschrift für Angewandte Mathematik und Physik, 2012, 64 (2), pp.223-251. 10.1007/s00033-012-0232-x . hal-00647798

\section{HAL Id: hal-00647798 \\ https://hal.inria.fr/hal-00647798}

Submitted on 2 Dec 2011

HAL is a multi-disciplinary open access archive for the deposit and dissemination of scientific research documents, whether they are published or not. The documents may come from teaching and research institutions in France or abroad, or from public or private research centers.
L'archive ouverte pluridisciplinaire HAL, est destinée au dépôt et à la diffusion de documents scientifiques de niveau recherche, publiés ou non, émanant des établissements d'enseignement et de recherche français ou étrangers, des laboratoires publics ou privés. 


\title{
On entropy weak solutions of Hughes' model for pedestrian motion
}

\author{
Nader El-Khatib, Paola Goatin and Massimiliano D. Rosini
}

\begin{abstract}
We consider a generalized version of Hughes' macroscopic model for crowd motion in the one-dimensional case. It consists in a scalar conservation law accounting for the conservation of the number of pedestrians, coupled with an eikonal equation giving the direction of the flux depending on pedestrian density. As a result of this non-trivial coupling, we have to deal with a conservation law with space-time discontinuous flux, whose discontinuity depends non-locally on the density itself. We propose a definition of entropy weak solution, which allows us to recover a maximum principle. Moreover, we study the structure of the solutions to Riemann-type problems and we construct them explicitly for small times, depending on the choice of the running cost in the eikonal equation. In particular, aiming at the optimization of the evacuation time, we propose a strategy that is optimal in the case of high densities. All results are illustrated by numerical simulations.
\end{abstract}

Mathematics Subject Classification (2010). Primary 35L65; Secondary 35F21, 90B20.

Keywords. Pedestrian flow, conservation laws, eikonal equation, weak entropy solutions, numerical approximations.

\section{Introduction}

We consider a generalization of the pedestrian flow model introduced by Hughes [10]. In the one-dimensional case, we consider the coupled equations

$$
\begin{aligned}
& \rho_{t}-\left(\rho v(\rho) \frac{\phi_{x}}{\left|\phi_{x}\right|}\right)_{x}=0, \\
& \left|\phi_{x}\right|=c(\rho),
\end{aligned}
$$

This research was supported by the ERC Starting Grant 2010 under the project "TRAffic Management by Macroscopic Models" and the Polonium 2011 (French-Polish cooperation program) under the project "CROwd Motion Modeling and Management". 
in the spatial domain $\Omega=]-1,1[$, together with homogeneous Dirichlet boundary conditions

$$
\begin{aligned}
& \rho(t,-1)=\rho(t, 1)=0, \\
& \phi(t,-1)=\phi(t, 1)=0 .
\end{aligned}
$$

Here $x \in \Omega$ is the space variable, $t \geq 0$ is the time, $\rho=\rho(t, x) \in[0,1]$ is the (normalized) crowd density, $c=c(\rho)$ is the running cost, $v(\rho)=(1-\rho)$ is the mean velocity and we set $f(\rho)=\rho v(\rho)=\rho(1-\rho)$. We assume that $c:[0,1] \rightarrow\left[1,+\infty\left[\right.\right.$ is a smooth function such that $c(0)=1$ and $c^{\prime}(\rho) \geq 0$. Recall that the literature usually proposes the choice

$$
c(\rho)=\frac{1}{v(\rho)}
$$

as in $9,10,11$.

Macroscopic models for pedestrian flow in one space dimension can be used to describe the evolution of a crowd moving along a corridor or a bridge. The first attempt to address this issue is represented by the paper of Colombo and Rosini [6], where the authors introduce non-classical shocks to account for the transition from normal to panic situations, see also [4, 5, 7, 15].

From the mathematical point of view, problem (1.1) presents a non trivial coupling between a scalar conservation law and an eikonal equation, that poses several challenging questions concerning existence, uniqueness, numerical approximation and construction of the solutions. In [9, the authors proved existence and uniqueness of solutions for a regularized version of equation (1.1b) with cost function (1.3), which in turn gives a smooth flux function for the conservation law (1.1a), and allows the use of classical techniques to prove existence and uniqueness by viscosity approximation and Kružkov entropy conditions [13, Equation (2.1)].

Here we are interested in weak solutions of the (non-regularized) hyperbolic problem (1.1). Observe that (1.1a) can be rewritten as

$$
\rho_{t}-\left(f(\rho) \operatorname{sgn}\left(\phi_{x}\right)\right)_{x}=0,
$$

and the unique viscosity solution to the Dirichlet problem (1.1b), (1.2b) is given by the value function of the corresponding control problem with discontinuous running cost $c(\rho)$, i.e.

$$
\phi(t, x)= \begin{cases}\int_{-1}^{x} c(\rho(t, y)) d y & \text { if } x \leq \xi(t), \\ \int_{x}^{1} c(\rho(t, y)) d y & \text { if } x \geq \xi(t),\end{cases}
$$

where $\xi(t)$ is implicitly defined by the identity

$$
\int_{-1}^{\xi(t)} c(\rho(t, y)) d y=\int_{\xi(t)}^{1} c(\rho(t, y)) d y .
$$

(We refer the reader to [16] for a proof of existence of a unique viscosity solution for Hamilton-Jacobi equations with discontinuous coefficients under 
appropriate compatibility conditions, here satisfied for example if $\rho(t, \cdot)$ is a function of bounded variation, see Section 6 for further details.) Therefore, equation 1.1a can be written as a scalar conservation law with discontinuous space-time dependent flux:

$$
\rho_{t}+F(t, x, \rho)_{x}=0
$$

where $F(t, x, \rho)=\operatorname{sgn}(x-\xi(t)) f(\rho)$. Observe that the position of the discontinuity is not a priori fixed, as assumed in previous results on conservation laws with discontinuous fluxes (see for example [12] and references therein), but depends non-locally on $\rho$ itself. Nevertheless, we propose an entropy condition that allows us to recover some key properties of the solutions. In particular, we prove a maximum principle depending on the $\mathbf{L}^{\infty}$-norm of the initial datum that refines the one given in [9] for the regularized problem. Moreover, we analyze the structure of the solutions for simple, Riemann-like, initial data and general running cost $c(\rho)$.

The model (1.1), (1.2) can be applied to describe a crowd exiting a corridor or a bridge at both ends, where the cost function $c(\rho)$ can be interpreted as the strategy used by or imposed to the pedestrians to reach the exits, and therefore can be selected depending on the specific situation. Since $c(\rho)$ increases with $\rho$, people will be led to avoid high density regions due to the corresponding augmentation of travel time. Aiming at the optimization of evacuation times, we investigate its dependence on the choice of the cost function $c(\rho)$, and we propose a strategy that is optimal in the case of high densities. All results are illustrated by numerical simulations.

Recently, a related approach has been introduced independently in 11. Nonetheless, in this paper the authors restrict the study to the choice (1.3), give a different definition of entropy solution, and concentrate on the study of the structure of the solutions to Riemann-type problems. Therefore, even if some results superpose, the strategies and aims are different.

The paper is organized as follows. In Section 2, we define the entropy condition and we derive the related properties of entropic solutions, among which the maximum principle. In Section 3, we detail the structure of the solution for Riemann-type initial data. Section 4 deals with the optimization of the evacuation time depending on the choice of the cost function. Numerical simulations illustrating the previous results are presented in Section 5. Finally, Section 6] collects some results concerning the eikonal equation (1.1b).

\section{Entropy conditions}

We consider the system

$$
\begin{aligned}
& \rho_{t}+F(t, x, \rho)_{x}=0, \quad x \in \Omega, t>0, \\
& \left|\phi_{x}\right|=c(\rho),
\end{aligned}
$$

with initial condition

$$
\rho(0, x)=\rho_{0}(x) \in[0,1],
$$


and (weak) boundary conditions (1.2). We start by defining a weak solution of (2.1) as a function $\rho \in \mathbf{L}^{\infty}\left(\mathbb{R}^{+} \times \Omega\right)$ such that for every $\psi \in \mathbf{C}_{\mathbf{c}}^{\infty}(\mathbb{R} \times \Omega)$ the following equality holds:

$$
\int_{0}^{+\infty} \int_{-1}^{1}\left(\rho \psi_{t}+F(t, x, \rho) \psi_{x}\right) d x d t+\int_{-1}^{1} \rho_{0}(x) \psi(0, x) d x=0 .
$$

If moreover we assume that $\rho \in \mathbf{C}^{\mathbf{0}}\left(\mathbb{R}^{+} ; \mathrm{BV}(\Omega) \cap \mathbf{L}^{\mathbf{1}}(\Omega)\right), \rho$ is a weak solution of (2.1) if and only if it satisfies in the weak sense

$$
\begin{array}{ll}
\rho_{t}-(f(\rho))_{x}=0, & x \in]-1, \xi(t)[, t>0, \\
\rho_{t}+(f(\rho))_{x}=0, & x \in] \xi(t), 1[, t>0, \\
\rho(0, x)=\rho_{0}(x), & x \in]-1,1[,
\end{array}
$$

where $\xi(t) \in \mathbf{C}^{\mathbf{0}}\left(\mathbb{R}^{+}\right.$) (see Lemma 6.1 in Section 6) is implicitly defined by (1.6), and the Rankine-Hugoniot condition

$$
f\left(\rho^{+}\right)+f\left(\rho^{-}\right)=\xi^{\prime}(t)\left(\rho^{+}-\rho^{-}\right)
$$

holds, where we have denoted by $\rho^{+}=\rho^{+}(t)=\rho(t, \xi(t)+)$ and $\rho^{-}=\rho^{-}(t)=$ $\rho(t, \xi(t)-)$ the right and left traces of $\rho$ at $x=\xi(t)$ (in the rest of the paper we will drop the time variable whenever clear from the context).

We remark here that if $\rho(t, \cdot)$ is continuous at $x=\xi(t)$, then (2.4) gives $f(\rho(t, \xi(t)))=0$ and therefore $\rho(t, \xi(t)) \in\{0,1\}$. Moreover, if $\rho(t, \xi(t))=1$, we have $\operatorname{sgn}(x-\xi(t)) f^{\prime}(\rho)<0$ and, by generalized backward characteristics (see [8]), we infer that $\left\|\rho_{0}\right\|_{\mathbf{L}^{\infty}(\Omega)}=1$.

Starting from entropy conditions for conservation laws with discontinuous flux functions, see [12, Definition 5.1] and [14, we give the following

Definition 2.1. (Entropy Condition). A weak solution $\rho$ of the initial-boundary value problem (2.1), (1.2) is an entropy weak solution if the following Kružkovtype entropy inequality holds for all $k \in \mathbb{R}$ and all test functions $\psi \in$ $\mathbf{C}_{\mathbf{c}}^{\infty}(\mathbb{R} \times \Omega), \psi \geq 0$ :

$$
\begin{aligned}
0 \leq & \int_{0}^{+\infty} \int_{-1}^{1}\left(|\rho-k| \psi_{t}+\Phi(t, x, \rho, k) \psi_{x}\right) d x d t+\int_{-1}^{1}\left|\rho_{0}(x)-k\right| \psi(0, x) d x \\
& +\operatorname{sgn}(k) \int_{0}^{+\infty}(f(\rho(t, 1-))-f(k)) \psi(t, 1) d t \\
& +\operatorname{sgn}(k) \int_{0}^{+\infty}(f(\rho(t,-1+))-f(k)) \psi(t,-1) d t \\
& +2 \int_{0}^{+\infty} f(k) \psi(t, \xi(t)) d t .
\end{aligned}
$$

Above, we have used the notation

$$
\Phi(t, x, \rho, k)=\operatorname{sgn}(\rho-k)(F(t, x, \rho)-F(t, x, k))
$$

and the entropy boundary condition introduced by Bardos et al. in [3]. In particular, the strong traces of the solution at the boundary points exist due to genuine non-linearity of the flux (see Vasseur [18]) and must satisfy

$$
f(\rho(t, \pm 1 \mp)) \geq f(k), \quad \text { for all } k \in[0, \rho(t, \pm 1 \mp)] .
$$


This in particular implies $\rho(t, \pm 1 \mp) \leq 1 / 2$.

Corollary 2.2. Let $\rho \in \mathbf{C}^{\mathbf{0}}\left(\mathbb{R}^{+} ; B V(\Omega) \cap \mathbf{L}^{\mathbf{1}}(\Omega)\right)$ be an entropy weak solution in the sense of Definition 2.1, then the following entropy jump condition must hold for all $k \in \mathbb{R}$ and a.e. $t \in \mathbb{R}^{+}$:

$$
\begin{aligned}
& \operatorname{sgn}\left(\rho^{+}(t)-k\right)\left(f\left(\rho^{+}(t)\right)-f(k)\right)-\xi^{\prime}(t)\left|\rho^{+}(t)-k\right| \\
+ & \operatorname{sgn}\left(\rho^{-}(t)-k\right)\left(f\left(\rho^{-}(t)\right)-f(k)\right)+\xi^{\prime}(t)\left|\rho^{-}(t)-k\right| \\
\leq & 2 f(k) .
\end{aligned}
$$

Remark 2.3. Taking $k \leq \rho^{-}, \rho^{+}$in (2.6) we obtain

$$
f\left(\rho^{+}\right)+f\left(\rho^{-}\right) \leq 4 f(k)+\xi^{\prime}(t)\left(\rho^{+}-\rho^{-}\right) ;
$$

while taking $k \geq \rho^{-}, \rho^{+},(2.6)$ gives

$$
f\left(\rho^{+}\right)+f\left(\rho^{-}\right) \geq \xi^{\prime}(t)\left(\rho^{+}-\rho^{-}\right) .
$$

In particular, we recover (2.4) by taking $k=0$ in (2.7). Finally, for $k$ between $\rho^{-}$and $\rho^{+}$we get

$$
\operatorname{sgn}\left(\rho^{+}-\rho^{-}\right)\left[f\left(\rho^{+}\right)-f\left(\rho^{-}\right)+\xi^{\prime}(t)\left(2 k-\rho^{+}-\rho^{-}\right)\right] \leq 2 f(k) .
$$

Proposition 2.4. Let $\rho \in \mathbf{C}^{\mathbf{0}}\left(\mathbb{R}^{+} ; B V(\Omega) \cap \mathbf{L}^{\mathbf{1}}(\Omega)\right)$ be an entropy weak solution in the sense of Definition [2.1 and assume $\rho^{+}(t) \neq \rho^{-}(t)$. Then the characteristic speeds at $x=\xi(t)$ must enter the switch curve $x=\xi(t)$ on the side of higher density, i.e.

$$
\begin{aligned}
f^{\prime}\left(\rho^{+}(t)\right) & \leq \xi^{\prime}(t), \text { if } \rho^{-}(t)<\rho^{+}(t), \\
-f^{\prime}\left(\rho^{-}(t)\right) & \geq \xi^{\prime}(t), \text { if } \rho^{-}(t)>\rho^{+}(t) .
\end{aligned}
$$

Proof. By multiplying (2.8) by $\left|\rho^{+}-\rho^{-}\right|$and using (2.4) we obtain that

$$
f\left(\rho^{+}\right)\left(k-\rho^{-}\right)-f\left(\rho^{-}\right)\left(\rho^{+}-k\right) \leq f(k)\left|\rho^{+}-\rho^{-}\right| .
$$

For $\rho^{-} \leq k \leq \rho^{+}$we get from (2.9) that

$$
\frac{f\left(\rho^{+}\right)-f(k)}{\rho^{+}-k} \leq \frac{f(k)+f\left(\rho^{-}\right)}{k-\rho^{-}} .
$$

Then, letting $k \nearrow \rho^{+}$and using (2.4) we get $\xi^{\prime}(t) \geq f^{\prime}\left(\rho^{+}(t)\right)$, hence characteristic lines are entering the sign change line $x=\xi(t)$ on the right hand side.

For $\rho^{+} \leq k \leq \rho^{-}$we get from (2.9) that

$$
\frac{f\left(\rho^{-}\right)-f(k)}{\rho^{-}-k} \leq \frac{f(k)+f\left(\rho^{+}\right)}{k-\rho^{+}} .
$$

Then, letting $k \nearrow \rho^{-}$and using (2.4) we get $\xi^{\prime}(t) \leq-f^{\prime}\left(\rho^{-}(t)\right)$, hence characteristic lines are entering the sign change line $x=\xi(t)$ on the left hand side. 
Incidentally, we observe that the definition of entropy weak solution implies that the traces at $x=\xi(t+)$ must satisfy the bounds $0 \leq \rho(t+, \xi(t+) \pm) \leq$ $\sup \{\rho(t, y): y \in]-1,1[\}$. Combining this with the classical maximum principle satisfied by the conservation laws (2.3) in the sectors $\left.\mathbb{R}^{+} \times\right]-1, \xi(t)[$ and $\left.\mathbb{R}^{+} \times\right] \xi(t), 1[$ respectively, we deduce the following Corollary.

Corollary 2.5 (Maximum principle). Let $\rho \in \mathbf{C}^{\mathbf{0}}\left(\mathbb{R}^{+} ; B V(\Omega) \cap \mathbf{L}^{\mathbf{1}}(\Omega)\right)$ be an entropy weak solution of (2.1), (1.2), then

$$
0 \leq \rho(t, x) \leq\left\|\rho_{0}\right\|_{\mathbf{L}^{\infty}(\Omega)} .
$$

Remark that by the above maximum principle, we can avoid blow up of cost functions like (1.3) by taking initial data $\rho_{0}(x) \in[0,1-\delta]$, for $\delta>0$.

In the next sections we will restrict the study to the case of Riemannlike initial data. Existence and uniqueness of solutions for general initial data go beyond the scope of the present article and will be the object of future study.

\section{The Riemann problem on an interval}

For later use, we denote by $t_{1}=t_{1}\left(\rho_{l}, \rho_{R}\right)>0$ the first interaction time between two waves, or a wave with the boundary or a wave with $x=\xi(t)$, and we introduce the notation $\sigma\left(\rho^{\prime}, \rho^{\prime \prime}\right)=\left(f\left(\rho^{\prime}\right)-f\left(\rho^{\prime \prime}\right)\right) /\left(\rho^{\prime}-\rho^{\prime \prime}\right)$ for the propagation speed of a shock between $\rho=\rho^{\prime}$ and $\rho=\rho^{\prime \prime}$ in the interval ]$\xi(t), 1[$.

We consider the sample initial-boundary value problem (2.1), (1.2) with initial data of the form

$$
\rho_{0}(x)= \begin{cases}\rho_{L} & \text { if } x<0 \\ \rho_{R} & \text { if } x>0 .\end{cases}
$$

First of all, we observe that by (1.6) we can easily compute the starting point $\xi_{0}=\xi(0)$ of the curve $\xi(t)$ :

$$
\xi_{0}= \begin{cases}\frac{1}{2}\left(1-\frac{c\left(\rho_{L}\right)}{c\left(\rho_{R}\right)}\right) \geq 0 & \text { if } \rho_{L}<\rho_{R}, \\ \frac{1}{2}\left(\frac{c\left(\rho_{R}\right)}{c\left(\rho_{L}\right)}-1\right) \leq 0 & \text { if } \rho_{L}>\rho_{R} .\end{cases}
$$

In the rest of the section we will restrict the study to $t \in\left[0, t_{1}[\right.$. The construction of solutions for times $t \geq t_{1}$ amounts to consider all the possible wave interactions, resulting in long computations, which are not necessary for our intents.

Lemma 3.1. The function $[t \mapsto \xi(t)]$ is linear in $] 0, t_{1}[$.

Proof. In order to prove that $\xi=\xi(t)$ is linear, it is enough to prove that the terms appearing in (1.6) are linear with respect to $t$. For $t \in] 0, t_{1}[$, the solution of the Riemann problem (1.2), (2.1), (3.1) performs only classical waves in $x \neq \xi(t)$, namely shocks and rarefactions. Therefore, the terms appearing in (1.6) are of the following types: 
1. Term related to a shock between values $\rho=\rho_{l}$ and $\rho=\rho_{r}$

$$
\int_{ \pm \sigma\left(\rho_{l}, \rho_{r}\right) t+x_{0}-\delta}^{ \pm \sigma\left(\rho_{l}, \rho_{r}\right) t+x_{0}} c\left(\rho_{l}\right) d x+\int_{ \pm \sigma\left(\rho_{l}, \rho_{r}\right) t+x_{0}}^{ \pm \sigma\left(\rho_{l}, \rho_{r}\right) t+x_{0}+\delta} c\left(\rho_{r}\right) d x=\left(c\left(\rho_{l}\right)+c\left(\rho_{r}\right)\right) \delta
$$

being $\delta \in \mathbb{R}^{+}$small enough.

2. Term related to a rarefaction in the domain $\Omega$ between values $\rho=\rho_{l}$ and $\rho=\rho_{r}$

$$
\int_{ \pm f^{\prime}\left(\rho_{l}\right) t+x_{0}}^{ \pm f^{\prime}\left(\rho_{r}\right) t+x_{0}} c\left(\left( \pm f^{\prime}\right)^{-1}\left(\frac{x-x_{0}}{t}\right)\right) d x=\mp 2 t \int_{\rho_{l}}^{\rho_{r}} c(\rho) d \rho .
$$

3. Term related to a rarefaction along $x=-1$ between the values $\rho=1 / 2$ and $\rho=\rho_{r}>1 / 2$

$$
\int_{-1}^{-f^{\prime}\left(\rho_{r}\right) t-1} c\left(\left(-f^{\prime}\right)^{-1}\left(\frac{x+1}{t}\right)\right) d x=2 t \int_{1 / 2}^{\rho_{r}} c(\rho) d \rho .
$$

4. Term related to a rarefaction along $x=1$ between the values $\rho=\rho_{l}>$ $1 / 2$ and $\rho=1 / 2$

$$
\int_{f^{\prime}\left(\rho_{l}\right) t+1}^{1} c\left(\left(f^{\prime}\right)^{-1}\left(\frac{x-1}{t}\right)\right) d x=-2 t \int_{\rho_{l}}^{1 / 2} c(\rho) d \rho .
$$

This proof is concluded.

The solution can be continuous or discontinuous along $x=\xi(t), t \in$ ] $0, t_{1}[$. These two possible cases will be considered separately in the following two subsections. We recall that, if the solution is continuous at $x=\xi(t)$, then $\rho^{-}(t)=\rho^{+}(t) \in\{0,1\}$. In this case, we apply equation (1.6) to explicitly compute $[t \mapsto \xi(t)], t \in] 0, t_{1}[$. On the other hand, if the solution is discontinuous at $x=\xi(t)$, then an intermediate state $\rho=\rho_{M}$ appears and is involved in the expression of the speed of propagation of $x=\xi(t)$ deduced from the RankineHugoniot condition (2.4). In this case, we use equation (1.6) to compute the intermediate value $\rho=\rho_{M}$. As a result we get the following theorem:

Theorem 3.2. For any initial data $\left(\rho_{L}, \rho_{R}\right) \in[0,1]^{2}$ and $\left.t \in\right] 0, t_{1}[$, the Riemann problem (1.2), (2.1), (3.1) admits a solution that is continuous along $x=\xi(t), t \in] 0, t_{1}[$, if and only if one of the following conditions hold (see figures 1 and [2):

1. $\rho_{L}, \rho_{R}>1 / 2$.

2. $\rho_{L}, \rho_{R} \leq 1 / 2$ and

$\rho_{R}-1<\int_{\rho_{R}}^{1 / 2}\left(c(\rho)-c\left(\rho_{R}\right)\right) d \rho-\int_{\rho_{L}}^{1 / 2}\left(c(\rho)-c\left(\rho_{L}\right)\right) d \rho<1-\rho_{L}$.

3. $\rho_{R} \leq 1 / 2<\rho_{L}$ and

$$
\int_{\rho_{R}}^{1 / 2}\left(c(\rho)-c\left(\rho_{R}\right)\right) d \rho<1-\rho_{L} .
$$



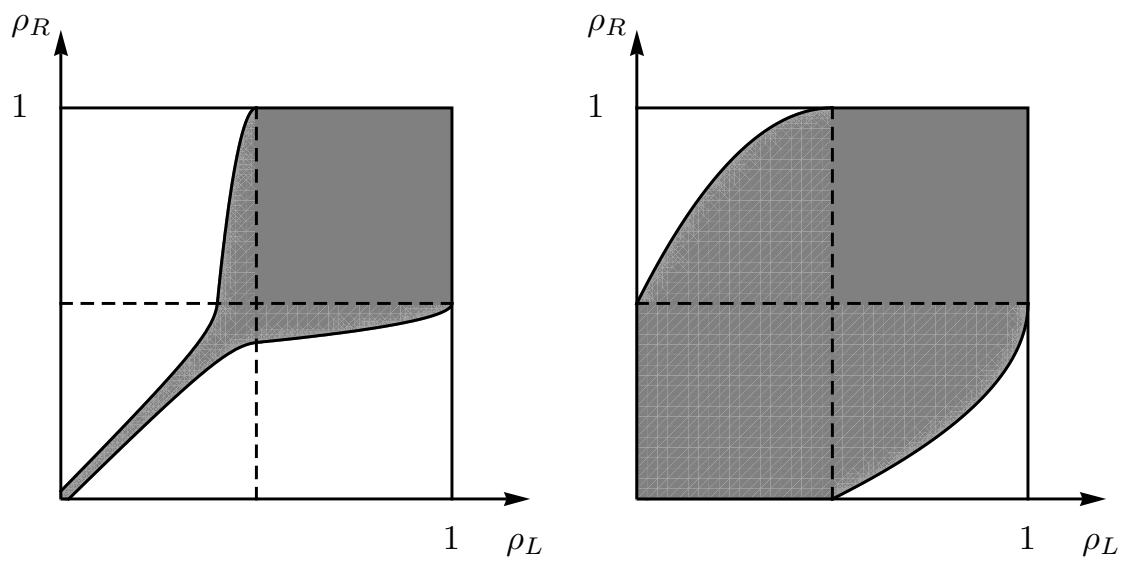

FiguRE 1. In both diagrams, the shaded areas represent the initial data for which the solution to the Riemann problem (1.2), (2.1), 3.1) is continuous along $x=\xi(t), t \in] 0, t_{1}[$. The left one corresponds to the cost function $c(\rho)=1+100 \rho$ and the right one to $c(\rho)=1+4 \rho$.
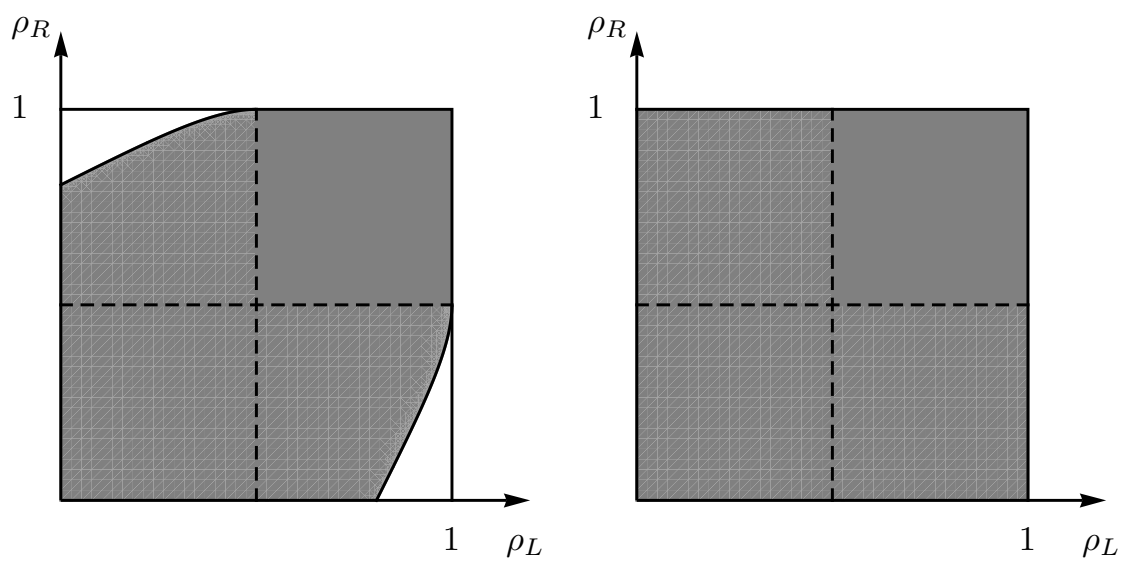

FiguRE 2. In both diagrams, the shaded areas represent the initial data for which the solution to the Riemann problem (1.2), (2.1), (3.1) is continuous along $x=\xi(t), t \in] 0, t_{1}[$. The left one corresponds to the cost function $c(\rho)=1 / v(\rho)$ and the right one to $c(\rho)=c_{o}(\rho)$ given by (4.6).

4. $\rho_{L} \leq 1 / 2<\rho_{R}$ and

$$
\int_{\rho_{L}}^{1 / 2}\left(c(\rho)-c\left(\rho_{L}\right)\right) d \rho<1-\rho_{R} .
$$


In all the other cases, the solution is discontinuous along $x=\xi(t), t \in] 0, t_{1}[$.

The details of the proof are given in the following subsections 3.1 and 3.2 . Due to the spatial symmetry of the problem, we will restrict our analysis to the case $1 \geq \rho_{L}>\rho_{R} \geq 0$. In this case, we have $\xi_{0}<0$ and the rarefaction wave exiting $x=0$ takes the following expression:

$$
\rho(t, x)= \begin{cases}\rho_{L} & \text { if } x<\left(1-2 \rho_{L}\right) t, \\ \frac{1}{2}\left(1-\frac{x}{t}\right) & \text { if }\left(1-2 \rho_{L}\right) t \leq x \leq\left(1-2 \rho_{R}\right) t, \\ \rho_{R} & \text { if } x>\left(1-2 \rho_{R}\right) t .\end{cases}
$$

\subsection{Solutions continuous along $x=\xi(t)$}

We study first the case $\rho_{L}=1$ (and $c(1)$ bounded).

- $\rho_{R} \leq 1 / 2$ : In this case, see Figure 3, left, a rarefaction enters the left boundary $x=-1$ and the equation (1.6) writes

$$
\begin{aligned}
& 2 t \int_{1 / 2}^{1} c(\rho) d \rho+c(1)\left(\xi(t)+f^{\prime}(1) t+1\right) \\
= & c(1)\left(f^{\prime}(1) t-\xi(t)\right)-2 t \int_{1}^{\rho_{R}} c(\rho) d \rho+c\left(\rho_{R}\right)\left(1-f^{\prime}\left(\rho_{R}\right) t\right),
\end{aligned}
$$

which gives

$$
2 t \int_{1 / 2}^{\rho_{R}} c(\rho) d \rho+c(1)(2 \xi(t)+1)-c\left(\rho_{R}\right)\left(1-f^{\prime}\left(\rho_{R}\right) t\right)=0
$$

i.e.

$$
\xi(t)=\xi_{0}+\frac{t}{c(1)}\left(\int_{\rho_{R}}^{1 / 2} c(\rho) d \rho-c\left(\rho_{R}\right)\left(\frac{1}{2}-\rho_{R}\right)\right) .
$$

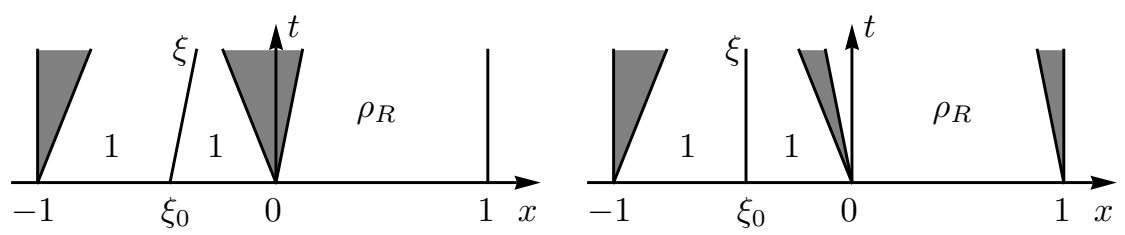

Figure 3. Solutions to the Riemann problem (1.2), (2.1), (3.1) corresponding to $\rho_{L}=1$ and $1 / 2 \geq \rho_{R}$, left, and $\rho_{R}>$ $1 / 2$, right, for $t \in] 0, t_{1}[$. The shaded areas correspond to rarefactions and the line to $x=\xi(t)$.

- $\rho_{R}>1 / 2$ : In this last case, see Figure 3 right, rarefaction waves appear at both the boundaries $x= \pm 1$ and the equation (1.6) writes

$$
\begin{aligned}
& 2 t \int_{1 / 2}^{1} c(\rho) d \rho+c(1)\left(\xi(t)+f^{\prime}(1) t+1\right) \\
= & c(1)\left(f^{\prime}(1) t-\xi(t)\right)-2 t \int_{1}^{\rho_{R}} c(\rho) d \rho+c\left(\rho_{R}\right)-2 t \int_{\rho_{R}}^{1 / 2} c(\rho) d \rho,
\end{aligned}
$$


which gives

$$
(2 \xi(t)+1) c(1)=c\left(\rho_{R}\right)
$$

i.e.

$$
\xi(t)=\xi_{0} .
$$

Assume now that $\rho_{L}<1$ and that the solution is continuous along $x=\xi(t), t \in] 0, t_{1}\left[\right.$, namely that $\rho^{-} \equiv 0 \equiv \rho^{+}$.

- $1 / 2 \geq \rho_{L}>\rho_{R}$ : In this case, characteristics are exiting the domain on both sides of $\xi_{0}$, and we expect to have two shocks leaving $\xi_{0}$, one moving with negative speed $\sigma_{-}=\rho_{L}-1$, and another moving with positive speed $\sigma_{+}=1-\rho_{L}$ (see Figure 4, left). In order to have existence of an entropy admissible solution continuous along $x=\xi(t)$, the following condition has to be satisfied

$$
\left.\xi_{0}+\sigma_{-} t<\xi(t)<\xi_{0}+\sigma_{+} t, \quad t \in\right] 0, t_{1}[.
$$

The rarefaction wave exiting $x=0$ is moving with positive speed. Then, for $t<t_{1}$, the position of the sign change of $\phi_{x}$ is given implicitly by the following equality

$$
\begin{aligned}
& c\left(\rho_{L}\right)\left(\sigma_{-} t+\xi_{0}+1\right)+c(0)\left(\xi(t)-\sigma_{-} t-\xi_{0}\right)=c(0)\left(\sigma_{+} t+\xi_{0}-\xi(t)\right) \\
& +c\left(\rho_{L}\right)\left(f^{\prime}\left(\rho_{L}\right) t-\sigma_{+} t-\xi_{0}\right)-2 t \int_{\rho_{L}}^{\rho_{R}} c(\rho) d \rho+c\left(\rho_{R}\right)\left(1-f^{\prime}\left(\rho_{R}\right) t\right)
\end{aligned}
$$

i.e.

$$
\begin{aligned}
\xi(t) & =\xi_{0}+\frac{c\left(\rho_{R}\right)}{2}\left(1-f^{\prime}\left(\rho_{R}\right) t\right)+\frac{c\left(\rho_{L}\right)}{2}\left(f^{\prime}\left(\rho_{L}\right) t-2 \xi_{0}-1\right)+t \int_{\rho_{R}}^{\rho_{L}} c(\rho) d \rho \\
& =\xi_{0}+t\left[c\left(\rho_{R}\right)\left(\rho_{R}-\frac{1}{2}\right)+c\left(\rho_{L}\right)\left(\frac{1}{2}-\rho_{L}\right)+\int_{\rho_{R}}^{\rho_{L}} c(\rho) d \rho\right] .
\end{aligned}
$$

Hence condition (3.6) gives

$$
\int_{\rho_{R}}^{1 / 2}\left(c(\rho)-c\left(\rho_{R}\right)\right) d \rho-\int_{\rho_{L}}^{1 / 2}\left(c(\rho)-c\left(\rho_{L}\right)\right) d \rho<1-\rho_{L} .
$$

Note that in the present case $\left(1 / 2 \geq \rho_{L}>\rho_{R}\right)$ the left hand side of the above inequality is non negative.
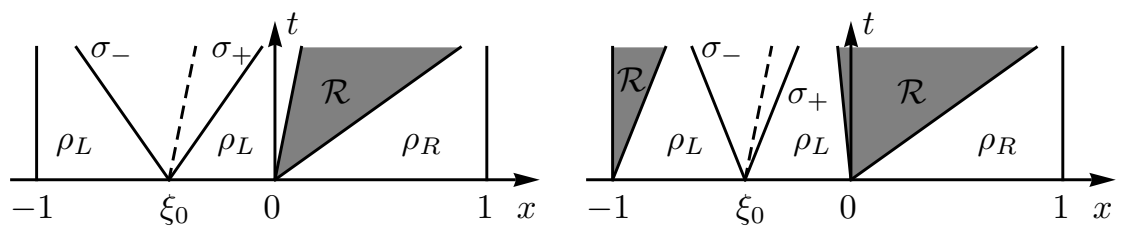

Figure 4. The solution to the Riemann problem (1.2), (2.1), (3.1) in the case $1 / 2 \geq \rho_{L}>\rho_{R}$, left, and $\rho_{L}>1 / 2 \geq$ $\rho_{R}$, right. The lines represent the shocks, the shaded areas the rarefactions and the dotted lines $x=\xi(t)$. 
- $\rho_{L}>1 / 2 \geq \rho_{R}$ : In this case, boundary conditions at $x=-1$ imply that a rarefaction wave forms between $\rho(t,-1+)=1 / 2$ and $\rho_{L}$, i.e.

$$
\rho(t, x)= \begin{cases}\frac{x+1}{2 t}+\frac{1}{2} & \text { if }-1<x \leq\left(2 \rho_{L}-1\right) t-1, \\ \rho_{L} & \text { if } x>\left(2 \rho_{L}-1\right) t-1 .\end{cases}
$$

Moreover, the rarefaction wave exiting $x=0$ is transonic (see Figure 4 right). Hence, for $t \in] 0, t_{1}[$, the equation defining $\xi(t)$ should read:

$$
\begin{aligned}
& 2 t \int_{1 / 2}^{\rho_{L}} c(\rho) d \rho+c\left(\rho_{L}\right)\left(\sigma_{-} t+\xi_{0}+f^{\prime}\left(\rho_{L}\right) t+1\right)+c(0)\left(\xi(t)-\sigma_{-} t-\xi_{0}\right) \\
= & c(0)\left(\sigma_{+} t+\xi_{0}-\xi(t)\right)+c\left(\rho_{L}\right)\left(f^{\prime}\left(\rho_{L}\right) t-\sigma_{+} t-\xi_{0}\right) \\
& -2 t \int_{\rho_{L}}^{\rho_{R}} c(\rho) d \rho+c\left(\rho_{R}\right)\left(1-f^{\prime}\left(\rho_{R}\right) t\right)
\end{aligned}
$$

which gives

$$
\begin{aligned}
\xi(t) & =\xi_{0}+\frac{c\left(\rho_{R}\right)}{2}\left(2 \rho_{R}-1\right) t-t \int_{\rho_{L}}^{\rho_{R}} c(\rho) d \rho-t \int_{1 / 2}^{\rho_{L}} c(\rho) d \rho \\
& =\xi_{0}+t\left[c\left(\rho_{R}\right)\left(\rho_{R}-\frac{1}{2}\right)+\int_{\rho_{R}}^{1 / 2} c(\rho) d \rho\right] .
\end{aligned}
$$

In this case condition (3.6) implies

$$
\int_{\rho_{R}}^{1 / 2}\left(c(\rho)-c\left(\rho_{R}\right)\right) d \rho<1-\rho_{L} .
$$

Note that the left hand side of the above inequality is non negative. Letting $\rho_{L} \nearrow 1$ in (3.10), we can easily see that the above condition can not be satisfied unless $c(\rho) \equiv 1$ for $\rho \in[0,1 / 2]$. In particular, the solution can not be continuous at $x=\xi(t)$ for general $c$.

- $\rho_{L}>\rho_{R}>1 / 2$ : In this last case, a rarefaction wave appears at the boundary $x=1$ between $\rho_{R}$ and $\rho(t, 1-)=1 / 2$, i.e.

$$
\rho(t, x)= \begin{cases}\rho_{R} & \text { if } x<\left(1-2 \rho_{R}\right) t+1, \\ \frac{1-x}{2 t}+\frac{1}{2} & \text { if }\left(1-2 \rho_{R}\right) t+1 \leq x<1 .\end{cases}
$$

Moreover, the rarefaction wave exiting $x=0$ has negative speeds (see Figure 5]). Hence, for $t \in] 0, t_{1}[$, the equation defining $\xi(t)$ should be:

$$
\begin{aligned}
& 2 t \int_{1 / 2}^{\rho_{L}} c(\rho) d \rho+c\left(\rho_{L}\right)\left(\sigma_{-} t+\xi_{0}+f^{\prime}\left(\rho_{L}\right) t+1\right)+c(0)\left(\xi(t)-\sigma_{-} t-\xi_{0}\right) \\
= & c(0)\left(\sigma_{+} t+\xi_{0}-\xi(t)\right)+c\left(\rho_{L}\right)\left(f^{\prime}\left(\rho_{L}\right) t-\sigma_{+} t-\xi_{0}\right) \\
& -2 t \int_{\rho_{L}}^{\rho_{R}} c(\rho) d \rho+c\left(\rho_{R}\right)-2 t \int_{\rho_{R}}^{1 / 2} c(\rho) d \rho
\end{aligned}
$$


which gives

$$
\xi(t)=\xi_{0}-t \int_{1 / 2}^{\rho_{L}} c(\rho) d \rho-t \int_{\rho_{L}}^{\rho_{R}} c(\rho) d \rho+c\left(\rho_{R}\right)-t \int_{\rho_{R}}^{1 / 2} c(\rho) d \rho \equiv \xi_{0} .
$$

In this case, condition (3.6) is always satisfied.

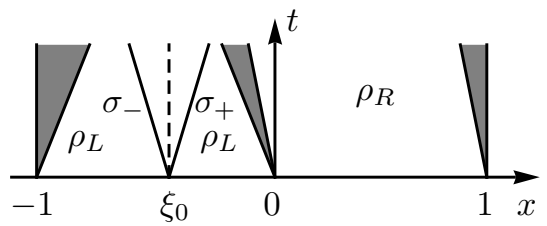

Figure 5. The solution to the Riemann problem (1.2), (2.1), 3.1) in the case $\rho_{L}>\rho_{R}>1 / 2$. The lines represent the shocks, the shaded areas the rarefactions and the dotted line $x=\xi(t)$.

We give now an example of initial data and cost function for which entropy weak solutions that are continuous at $x=\xi(t)$ do not exist. Let us take $c(\rho)=1 / v(\rho)$ and consider the Riemann problem with $\rho_{L}>1 / 2$ and $\rho_{R}=0$. In this case, equation (3.10) gives

$$
\ln 2-1 / 2<1-\rho_{L},
$$

i.e.

$$
\rho_{L}<3 / 2-\ln 2 \sim 0.8068528 .
$$

In Section 5.2. we show by numerical simulations that, taking $\rho_{L}>3 / 2-$ $\ln 2$ oscillations appear around $x=\xi(t)$, and a small group of people, that originally moved towards the right exit, changes direction and moves towards left. In particular, the traces of the solution at $x=\xi(t)$ are not equal to 0 .

\subsection{Solutions discontinuous along $x=\xi(t)$}

Assume that $\left\|\rho_{0}\right\|_{\mathbf{L}^{\infty}(\Omega)}<1$ and that the solution is discontinuous along $x=\xi(t)$. By Lemma 3.1, equations (2.4) and (3.2) we have for $t \in] 0, t_{1}[$

$$
\xi(t)=\frac{1}{2}\left(\frac{c\left(\rho_{R}\right)}{c\left(\rho_{L}\right)}-1\right)+\frac{f\left(\rho^{+}(t)\right)+f\left(\rho^{-}(t)\right)}{\rho^{+}(t)-\rho^{-}(t)} t .
$$

Furthermore, the method of characteristics ensures that one of the two trace values taken at $x=\xi(t)$ has to be $\rho=\rho_{L}$. Let $\rho=\rho_{M}$ be the other one. We observe that $\rho_{M}>\rho_{L}$ implies an incoherency in the wave speeds. Therefore, in accordance with the maximum principle, we must have $\rho_{M}<\rho_{L}$. As a consequence, $\xi^{\prime}(t) \neq 0$ for $\left.t \in\right] 0, t_{1}$ [ and two lines of discontinuity start from $x=\xi_{0}$ between the constant states $\rho=\rho_{L}, \rho=\rho_{M}$ and $\rho=\rho_{L}$. 

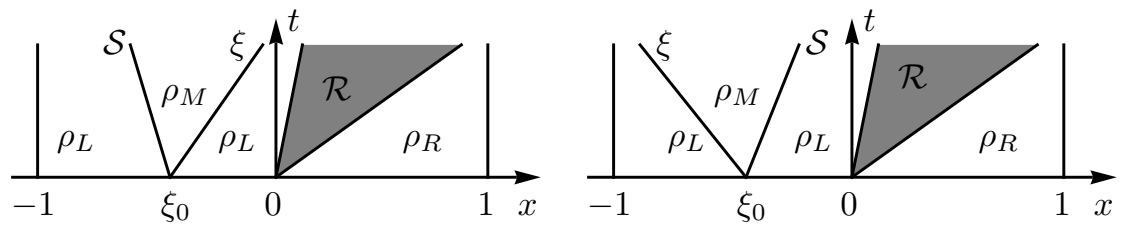

Figure 6. Solutions to the Riemann problem (1.2), (2.1), (3.1) corresponding to $1 / 2 \geq \rho_{L}>\rho_{R}$ and discontinuous at $x=\xi(t)$ with $\xi^{\prime}(t)>0$, left, and $\xi^{\prime}(t)<0$, right, for $t \in$ ] $0, t_{1}[$. The shaded areas correspond to rarefactions, denoted $\mathcal{R}$, and the lines to discontinuities, where $\mathcal{S}$ denote classical shocks.

- $1 / 2 \geq \rho_{L}>\rho_{R}:$ Assume that $\xi^{\prime}(t)>0$ for $\left.t \in\right] 0, t_{1}[$, see Figure 6, left. In this case $\rho^{-}(t)=\rho_{M}, \rho^{+}(t)=\rho_{L}$ and (1.6) writes

$$
\begin{aligned}
& \left(-\sigma\left(\rho_{M}, \rho_{L}\right) t+\xi_{0}+1\right) c\left(\rho_{L}\right)+\left(\xi(t)+\sigma\left(\rho_{M}, \rho_{L}\right) t-\xi_{0}\right) c\left(\rho_{M}\right) \\
= & \left(f^{\prime}\left(\rho_{L}\right) t-\xi(t)\right) c\left(\rho_{L}\right)-2 t \int_{\rho_{L}}^{\rho_{R}} c(\rho) d \rho+\left(1-f^{\prime}\left(\rho_{R}\right) t\right) c\left(\rho_{R}\right),
\end{aligned}
$$

or equivalently

$$
2\left(\mathcal{A}\left(\rho_{L}, \rho_{M}\right)-\mathcal{B}\left(\rho_{L}, \rho_{R}\right)\right) t=0
$$

where

$$
\begin{aligned}
\mathcal{A}\left(\rho_{L}, \rho_{M}\right) & =\frac{c\left(\rho_{M}\right) f\left(\rho_{L}\right)+c\left(\rho_{L}\right) f\left(\rho_{M}\right)}{\rho_{L}-\rho_{M}} \\
\mathcal{B}\left(\rho_{L}, \rho_{R}\right) & =\int_{\rho_{R}}^{1 / 2}\left(c(\rho)-c\left(\rho_{R}\right)\right) d \rho-\int_{\rho_{L}}^{1 / 2}\left(c(\rho)-c\left(\rho_{L}\right)\right) d \rho .
\end{aligned}
$$

Since the above equation needs to be satisfied for any $t \in] 0, t_{1}[$, we deduce

$$
\mathcal{A}\left(\rho_{L}, \rho_{M}\right)=\mathcal{B}\left(\rho_{L}, \rho_{R}\right) .
$$

Observe that for any fixed $\rho_{L} \leq 1 / 2$, the function $\left[\rho \mapsto \mathcal{A}\left(\rho_{L}, \rho\right)\right]$ is positive and increasing in $\left[0, \rho_{L}\left[\right.\right.$, with $\mathcal{A}\left(\rho_{L}, 0\right)=1-\rho_{L}$ and $\lim _{\rho} \nearrow_{L} \mathcal{A}\left(\rho_{L}, \rho\right)=+\infty$, while the function $\left[\rho \mapsto \mathcal{B}\left(\rho_{L}, \rho\right)\right]$ is positive and decreasing in $\left[0, \rho_{L}\right]$, with $B\left(\rho_{L}, \rho_{L}\right)=0$. Therefore, for any fixed $\rho_{L}, \rho_{R} \in\left[0,1 / 2\left[\right.\right.$ with $\rho_{R}<\rho_{L}$, the existence of $\rho_{M} \in\left[0, \rho_{L}\right.$ [ for which condition (3.14) holds is equivalent to require that

$$
\mathcal{B}\left(\rho_{L}, \rho_{R}\right) \geq \mathcal{A}\left(\rho_{L}, 0\right)
$$

namely

$$
\int_{\rho_{R}}^{1 / 2}\left(c(\rho)-c\left(\rho_{R}\right)\right) d \rho-\int_{\rho_{L}}^{1 / 2}\left(c(\rho)-c\left(\rho_{L}\right)\right) d \rho \geq 1-\rho_{L} .
$$

Moreover, the existence of $\rho_{M}, \rho_{R} \in\left[0, \rho_{L}\right.$ [ satisfying condition (3.14) is equivalent to require that

$$
\mathcal{B}\left(\rho_{L}, 0\right)-\mathcal{A}\left(\rho_{L}, 0\right)=\int_{0}^{\rho_{L}} c(\rho) d \rho+c\left(\rho_{L}\right)\left(\frac{1}{2}-\rho_{L}\right)+\rho_{L}-\frac{3}{2} \geq 0 .
$$


Above, the equality corresponds to the limit case $\rho_{M}=0=\rho_{R}$. Observing that $\partial_{\rho}(\mathcal{B}(\rho, 0)-\mathcal{A}(\rho, 0))=1-(\rho-1 / 2) c^{\prime}(\rho)>0$, we infer that $[\rho \mapsto$ $(\mathcal{B}(\rho, 0)-\mathcal{A}(\rho, 0))]$ is increasing in $[0,1 / 2]$. Therefore, necessary and sufficient condition to have the existence of a solution for (3.14) is that $\mathcal{B}(1 / 2,0)-$ $\mathcal{A}(1 / 2,0) \geq 0$, or equivalently

$$
\int_{0}^{1 / 2} c(\rho) d \rho \geq 1 .
$$

It remains to consider the case $\xi^{\prime}(t)<0$ for $\left.t \in\right] 0, t_{1}$ [, see Figure 6] right. In this case $\rho^{-}(t)=\rho_{L}, \rho^{+}(t)=\rho_{M}$ and proceeding as before, we obtain that (1.6) is equivalent to

$$
\mathcal{A}\left(\rho_{L}, \rho_{M}\right)+\mathcal{B}\left(\rho_{L}, \rho_{R}\right)=0,
$$

which is never satisfied for the above analysis.
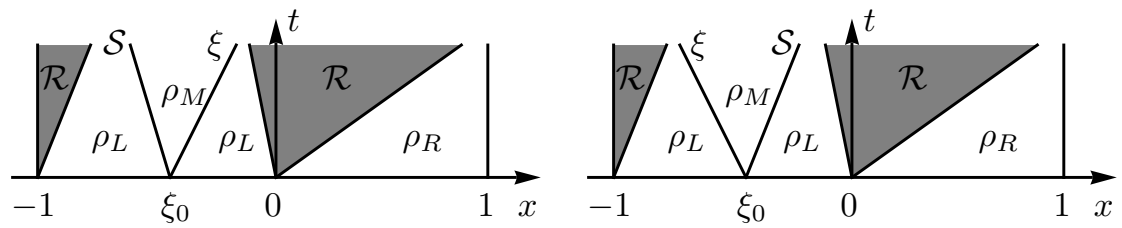

Figure 7. Solutions to the Riemann problem (1.2), (2.1), (3.1) corresponding to $\rho_{L}>1 / 2 \geq \rho_{R}$ and discontinuous at $x=\xi(t)$ with $\xi^{\prime}(t)>0$, left, and $\xi^{\prime}(t)<0$, right, for $t \in] 0, t_{1}[$. The shaded areas correspond to rarefactions and the lines to discontinuities.

- $\rho_{L}>1 / 2 \geq \rho_{R}:$ Assume that $\left.\xi^{\prime}(t)>0, t \in\right] 0, t_{1}[$, see Figure 7, left. In this case $\rho^{-}(t)=\rho_{M}, \rho^{+}(t)=\rho_{L}$ and (1.6) writes

$$
\begin{aligned}
& 2 t \int_{1 / 2}^{\rho_{L}} c(\rho) d \rho+\left(-\sigma\left(\rho_{M}, \rho_{L}\right) t+\xi_{0}+f^{\prime}\left(\rho_{L}\right) t+1\right) c\left(\rho_{L}\right) \\
& +\left(\xi(t)+\sigma\left(\rho_{M}, \rho_{L}\right) t-\xi_{0}\right) c\left(\rho_{M}\right) \\
= & \left(f^{\prime}\left(\rho_{L}\right) t-\xi(t)\right) c\left(\rho_{L}\right)-2 t \int_{\rho_{L}}^{\rho_{R}} c(\rho) d \rho+\left(1-f^{\prime}\left(\rho_{R}\right) t\right) c\left(\rho_{R}\right),
\end{aligned}
$$

or equivalently

$$
\left(\mathcal{A}\left(\rho_{L}, \rho_{M}\right)-\mathcal{B}\left(1 / 2, \rho_{R}\right)\right) t=0,
$$

where $\mathcal{A}$ and $\mathcal{B}$ are given by (3.13). Since the above equation needs to be satisfied for any $t \in] 0, t_{1}[$, we deduce

$$
\mathcal{A}\left(\rho_{L}, \rho_{M}\right)=\mathcal{B}\left(1 / 2, \rho_{R}\right) .
$$

For any fixed $\left.\rho_{L} \in\right] 1 / 2,1\left[\right.$, the function $\left[\rho \mapsto \mathcal{A}\left(\rho_{L}, \rho\right)\right]$ is positive in $\left[0, \rho_{L}[\right.$, with $\min _{\rho \in\left[0, \rho_{L}[\right.} \mathcal{A}\left(\rho_{L}, \rho\right)=\mathcal{A}\left(\rho_{L}, 0\right)=1-\rho_{L}$ and $\lim _{\rho} \rho_{L} \mathcal{A}\left(\rho_{L}, \rho\right)=+\infty$, while the function $[\rho \mapsto \mathcal{B}(1 / 2, \rho)]$ is positive and decreasing in $[0,1 / 2]$, with 
$B(1 / 2,1 / 2)=0$. Therefore, for any given $\rho_{L}, \rho_{R} \in\left[0,1\left[\right.\right.$ with $\rho_{R} \leq 1 / 2<\rho_{L}$, the existence of $\rho_{M} \in\left[0, \rho_{L}\right.$ [ satisfying (3.17] is equivalent to

$$
\mathcal{A}\left(\rho_{L}, 0\right) \leq \mathcal{B}\left(1 / 2, \rho_{R}\right)
$$

namely

$$
\int_{\rho_{R}}^{1 / 2}\left(c(\rho)-c\left(\rho_{R}\right)\right) d \rho \geq 1-\rho_{L} .
$$

Moreover, for any fixed $\left.\rho_{L} \in\right] 1 / 2,1\left[\right.$, the existence of $\rho_{M}, \rho_{R} \in\left[0, \rho_{L}[\right.$ satisfying condition (3.17) is equivalent to

$$
\mathcal{B}(1 / 2,0)-\mathcal{A}\left(\rho_{L}, 0\right)=\int_{0}^{1 / 2} c(\rho) d \rho+\rho_{L}-\frac{3}{2} \geq 0 .
$$

Above, the equality corresponds to the case $\rho_{M}=0=\rho_{R}$. By observing that $[\rho \mapsto(\mathcal{B}(1 / 2,0)-\mathcal{A}(\rho, 0))]$ is increasing in $] 1 / 2,1[$, we finally obtain that sufficient condition to have the existence of a solution for (3.17) is that $\lim _{\rho \nearrow_{1}} \mathcal{B}(1 / 2,0)-\mathcal{A}(\rho, 1) \geq 0$, namely

$$
\int_{0}^{1 / 2} c(\rho) d \rho \geq \frac{1}{2}
$$

It remains to consider the case $\xi^{\prime}(t)<0$ for $\left.t \in\right] 0, t_{1}[$, see Figure 7 . right. In this case $\rho^{-}(t)=\rho_{L}, \rho^{+}(t)=\rho_{M}$ and proceeding as before, we obtain that (1.6) is equivalent to

$$
\mathcal{A}\left(\rho_{L}, \rho_{M}\right)+\mathcal{B}\left(1 / 2, \rho_{R}\right)=0,
$$

which is never satisfied for the above analysis.
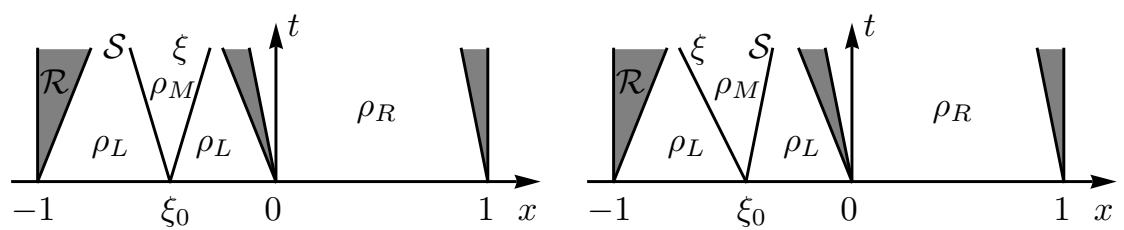

Figure 8. Solutions to the Riemann problem (1.2), (2.1), (3.1) corresponding to $\rho_{L}>\rho_{R}>1 / 2$ and discontinuous at $x=\xi(t)$ with $\xi^{\prime}(t)>0$, left, and $\xi^{\prime}(t)<0$, right, for $t \in] 0, t_{1}[$. The shaded areas correspond to rarefactions and the lines to discontinuities.

- $\rho_{L}>\rho_{R}>1 / 2$ : If $\xi^{\prime}(t)>0$ for $\left.t \in\right] 0, t_{1}[$, see Figure [8, left, then $\rho^{-}(t)=\rho_{M}, \rho^{+}(t)=\rho_{L}$ and (1.6) writes

$$
\begin{aligned}
& \quad 2 t \int_{1 / 2}^{\rho_{L}} c(\rho) d \rho+c\left(\rho_{L}\right)\left(-\sigma\left(\rho_{L}, \rho_{M}\right) t+\xi_{0}+f^{\prime}\left(\rho_{L}\right) t+1\right) \\
& \quad+c\left(\rho_{M}\right)\left(\xi(t)+\sigma\left(\rho_{L}, \rho_{M}\right) t-\xi_{0}\right) \\
& =c\left(\rho_{L}\right)\left(f^{\prime}\left(\rho_{L}\right) t-\xi(t)\right)-2 t \int_{\rho_{L}}^{\rho_{R}} c(\rho) d \rho+c\left(\rho_{R}\right)-2 t \int_{\rho_{R}}^{1 / 2} c(\rho) d \rho
\end{aligned}
$$


or equivalently

$$
\mathcal{A}\left(\rho_{L}, \rho_{M}\right)=0
$$

which can never be satisfied. In the case $\xi^{\prime}(t)<0$ for $\left.t \in\right] 0, t_{1}[$, see Figure 8 , right, then $\rho^{-}(t)=\rho_{L}, \rho^{+}(t)=\rho_{M}$ and (1.6) writes

$$
\begin{aligned}
& 2 t \int_{1 / 2}^{\rho_{L}} c(\rho) d \rho+c\left(\rho_{L}\right)\left(\xi(t)+f^{\prime}\left(\rho_{L}\right) t+1\right) \\
= & c\left(\rho_{M}\right)\left(\sigma\left(\rho_{L}, \rho_{M}\right) t+\xi_{0}-\xi(t)\right)+c\left(\rho_{L}\right)\left(f^{\prime}\left(\rho_{L}\right) t-\sigma\left(\rho_{L}, \rho_{M}\right) t-\xi_{0}\right) \\
& -2 t \int_{\rho_{L}}^{\rho_{R}} c(\rho) d \rho+c\left(\rho_{R}\right)-2 t \int_{\rho_{R}}^{1 / 2} c(\rho) d \rho,
\end{aligned}
$$

or equivalently

$$
\mathcal{A}\left(\rho_{L}, \rho_{M}\right)=0
$$

which can never be satisfied.

\section{Optimization of the evacuation time}

In this section, we consider the problem of how to optimize the evacuation time by choosing a proper running cost $c$. In fact, $c=c(\rho)$ can be seen as the strategy pedestrians choose or are forced to use to evacuate the corridor, and therefore can be selected depending on the specific situation.

By definition, the global exit time is the maximum between the exit times through the left and right exits, namely

$$
T_{\text {exit }}=\max \left\{T_{l}, T_{r}\right\}
$$

Aiming at optimizing the evacuation strategy, we find natural to avoid situations in which pedestrians change direction along the way. Therefore, we restrict our attention to entropy weak solutions of the Riemann problem (2.1), (3.1), (1.2) which are continuous at $x=\xi(t)$. We claim that the minimum evacuation time corresponds to the case $T_{l}=T_{r}$. In fact, $\left[\xi(0) \mapsto T_{l}(\xi(0))\right]$ is a strictly increasing function, while $\left[\xi(0) \mapsto T_{r}(\xi(0))\right]$ is strictly decreasing. Assuming that $\rho_{L}>\rho_{R}$, the other case being analogous because of the symmetries of the problem, direct computations show that the condition $T_{l}(\xi(0))=T_{\text {exit }}=T_{r}(\xi(0))$ gives $\xi(0)=\xi_{o}\left(\rho_{L}, \rho_{R}\right)$ and $T_{\text {exit }}=T_{o}\left(\rho_{L}, \rho_{R}\right)$, where (see Figure 9, left) 

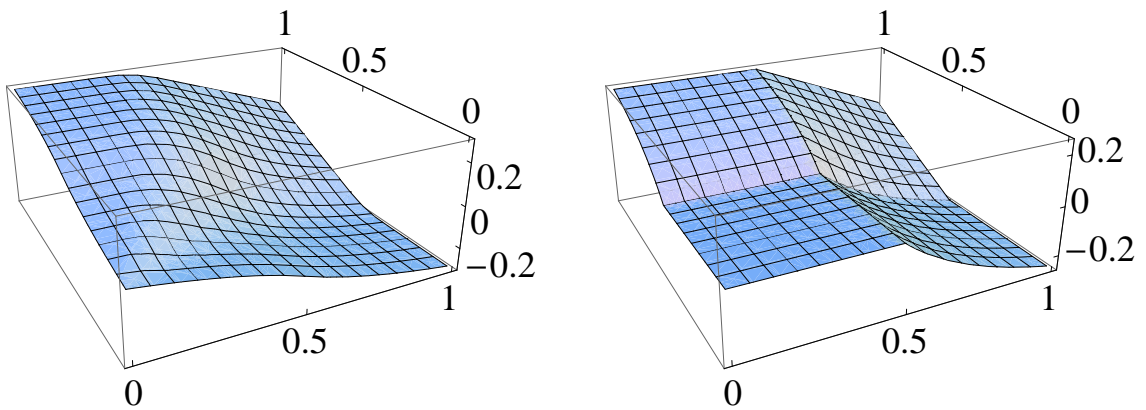

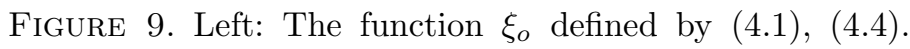
Right: The function $\xi_{0}^{c_{o}}$ defined by (4.7).

$$
\xi_{o}\left(\rho_{L}, \rho_{R}\right)= \begin{cases}-\frac{1}{2}\left(1-\frac{\rho_{R}}{\rho_{L}}\right) & \text { if }\left(\rho_{L}, \rho_{R}\right) \in A_{1}, \\ \frac{1-6 \rho_{L}+\sqrt{4 \rho_{L}^{2}+4 \rho_{L}-1}}{8 \rho_{L}} & \text { if }\left(\rho_{L}, \rho_{R}\right) \in A_{2}, \\ \frac{\left(1-4 \rho_{L}\left(1-\rho_{R}\right)\right) \rho_{R}}{\left(1+4 \rho_{R}\left(1-\rho_{R}\right)\right) \rho_{L}} & \text { if }\left(\rho_{L}, \rho_{R}\right) \in A_{3}, \\ -\frac{(\sqrt{2}-1) \rho_{L}}{1+\sqrt{2}-2 \rho_{L}} & \text { if }\left(\rho_{L}, \rho_{R}\right) \in A_{4}, \\ -\frac{\left(\rho_{L}-\rho_{R}\right) \rho_{R}}{\left(1-\rho_{L}\right) \rho_{L}+\left(1-\rho_{R}\right) \rho_{R}} & \text { if }\left(\rho_{L}, \rho_{R}\right) \in A_{5},\end{cases}
$$

and

$$
T_{o}\left(\rho_{L}, \rho_{R}\right)= \begin{cases}2\left(\rho_{L}+\rho_{R}\right) & \text { if }\left(\rho_{L}, \rho_{R}\right) \in A_{1}, \\ \frac{4\left(\rho_{L}+\rho_{R}\right)}{1+4 \rho_{R}\left(1-\rho_{R}\right)} & \text { if }\left(\rho_{L}, \rho_{R}\right) \in A_{2}, \\ \frac{1+2 \rho_{L}+\sqrt{4 \rho_{L}^{2}+4 \rho_{L}-1}}{2} & \text { if }\left(\rho_{L}, \rho_{R}\right) \in A_{3}, \\ \frac{\rho_{L}+\rho_{R}}{\rho_{L}\left(1-\rho_{L}\right)+\rho_{R}\left(1-\rho_{R}\right)} & \text { if }\left(\rho_{L}, \rho_{R}\right) \in A_{4}, \\ \frac{1+2(1+\sqrt{2}) \rho_{L}}{1+4\left(1-\rho_{L}\right) \rho_{L}} & \text { if }\left(\rho_{L}, \rho_{R}\right) \in A_{5} .\end{cases}
$$




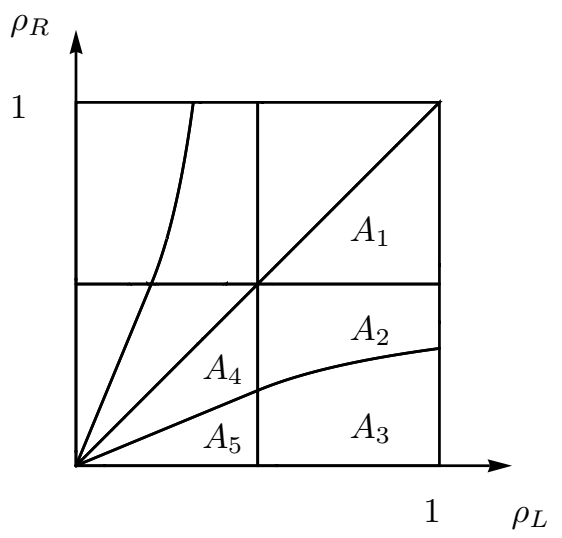

Figure 10. The subsets $A_{1}, \ldots, A_{5}$ of $[0,1]^{2}$ defined by (4.3).

The sets $A_{1}, \ldots, A_{5}$ above are defined by (see Figure (10)):

$$
\begin{aligned}
& A_{1}=\left\{\left(\rho_{L}, \rho_{R}\right) \in[0,1]^{2}: \frac{1}{2} \leq \rho_{R} \leq \rho_{L}\right\}, \\
& A_{2}=\left\{\left(\rho_{L}, \rho_{R}\right) \in[0,1]^{2}: \frac{\sqrt{4 \rho_{L}^{2}+4 \rho_{L}-1}}{2}-\rho_{L}<\rho_{R}<\frac{1}{2} \leq \rho_{L}\right\}, \\
& A_{3}=\left\{\left(\rho_{L}, \rho_{R}\right) \in[0,1]^{2}: \rho_{R}<\frac{\sqrt{4 \rho_{L}^{2}+4 \rho_{L}-1}}{2}-\rho_{L}, \frac{1}{2}<\rho_{L}\right\}, \\
& A_{4}=\left\{\left(\rho_{L}, \rho_{R}\right) \in[0,1]^{2}: \frac{1}{2}>\rho_{L} \geq \rho_{R}, \rho_{R}>(\sqrt{2}-1) \rho_{L}\right\}, \\
& A_{5}=\left\{\left(\rho_{L}, \rho_{R}\right) \in[0,1]^{2}: \rho_{R}<(\sqrt{2}-1) \rho_{L}, \rho_{L} \leq \frac{1}{2}\right\} .
\end{aligned}
$$

By symmetry, we easily recover

$$
\xi_{o}\left(\rho_{L}, \rho_{R}\right)=-\xi_{o}\left(\rho_{R}, \rho_{L}\right) .
$$

The resulting function $\xi_{o}:[0,1]^{2} \rightarrow \Omega$ is continuous and satisfies the bound $\left\|\xi_{o}\right\|_{\mathbf{C}^{\mathbf{o}}\left([0,1]^{2} ;[-1,1]\right)} \leq(5-\sqrt{7}) / 8 \sim 0.2942811$, see Figure 9, left.

We investigate the existence of a cost function corresponding to $\xi_{o}$ given by (4.1), (4.4). By choosing $\rho=\rho_{L}>\rho_{R}=0$ in (3.2) we obtain that

$$
\tilde{c}(\rho)=\frac{1}{1+2 \xi_{o}(\rho, 0)}= \begin{cases}\frac{4 \sqrt{2} \rho^{2}-2(1+2 \sqrt{2}) \rho+1}{8 \rho^{2}-8 \rho+1} & \text { if } \rho<\frac{1}{2}, \\ \frac{2 \rho\left(2 \rho-1+\sqrt{4 \rho^{2}+4 \rho-1}\right)}{4 \rho-1} & \text { if } \rho \geq \frac{1}{2} .\end{cases}
$$

Therefore $\tilde{c} \in \mathbf{C}^{\mathbf{1}}([0,1],[1,2(1+\sqrt{7}) / 3])$ is candidate to be a cost function that optimizes the evacuation time. Nevertheless, condition (3.2) is not satisfied for general initial data by $c=\tilde{c}$ defined by (4.5) and $\xi(0)=\xi_{o}$ defined 

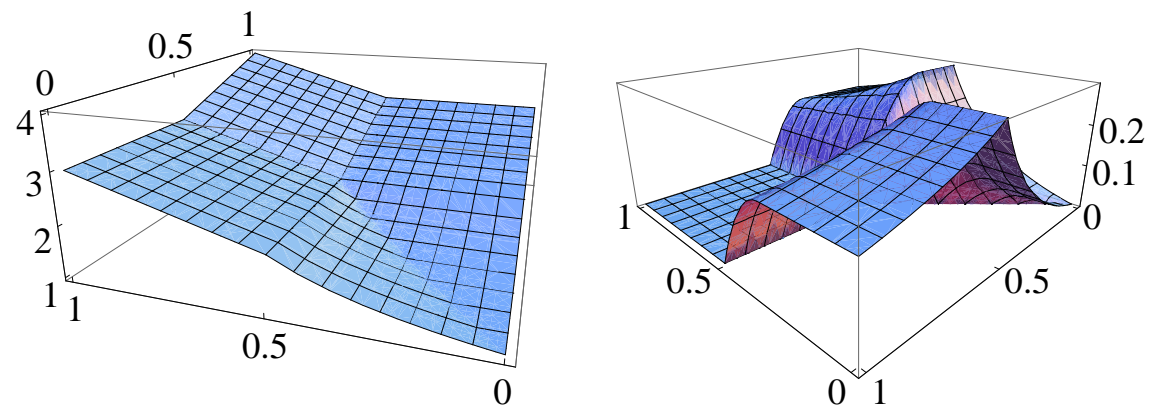

Figure 11. Left: The evacuation time $T_{\text {exit }}^{c_{o}}$ given by (4.8).

Right: $T_{\text {exit }}^{c_{o}}-T_{o}$, where $T_{o}$ is defined by (4.2).

by (4.1), (4.4). As a consequence, we infer that a cost function that optimizes the evacuation time for any initial data can not exist.

For the above considerations, it is natural to look for a cost function which optimizes the evacuation time in the case of high densities. By imposing condition (3.10), condition (3.2) with $\xi_{0}=\xi_{o}$ (defined by (4.1), (4.4)) for $\left(\rho_{L}, \rho_{R}\right) \in A_{1}$, and imposing continuity, we find the cost function

$$
c_{o}(\rho)= \begin{cases}1 & \text { if } \rho<1 / 2 \\ 2 \rho & \text { if } \rho \geq 1 / 2 .\end{cases}
$$

With this choice, by 3.2 we obtain that $\xi_{0}=\xi_{0}^{c_{o}}\left(\rho_{L}, \rho_{R}\right)$, where

$$
\xi_{0}^{c_{o}}\left(\rho_{L}, \rho_{R}\right)= \begin{cases}0 & \text { if } \rho_{L}, \rho_{R} \leq \frac{1}{2}, \\ \frac{1}{2}\left(1-\frac{1}{2 \rho_{R}}\right) & \text { if } \rho_{L} \leq \frac{1}{2} \leq \rho_{R}, \\ \frac{1}{2}\left(\frac{1}{2 \rho_{L}}-1\right) & \text { if } \rho_{R} \leq \frac{1}{2} \leq \rho_{L}, \\ \frac{1}{2}\left(1-\frac{\rho_{L}}{\rho_{R}}\right) & \text { if } \frac{1}{2} \leq \rho_{L} \leq \rho_{R}, \\ \frac{1}{2}\left(\frac{\rho_{R}}{\rho_{L}}-1\right) & \text { if } \frac{1}{2} \leq \rho_{R} \leq \rho_{L},\end{cases}
$$


see Figure 9] right. We observe that $\xi_{0}^{c_{o}}$ is a continuous function, satisfies (4.4) and $\left\|\xi_{0}^{c_{o}}\right\|_{\mathbf{C}^{\mathbf{o}}\left([0,1]^{2} ; \mathbb{R}\right)}=1 / 4$. The corresponding evacuation time is

$$
T_{\text {exit }}^{c_{o}}\left(\rho_{L}, \rho_{R}\right)= \begin{cases}\frac{1}{1-\rho_{L}} & \text { if } \rho_{R} \leq \rho_{L} \leq \frac{1}{2}, \\ \frac{1}{1-\rho_{R}} & \text { if } \rho_{L} \leq \rho_{R} \leq \frac{1}{2}, \\ 1+2 \rho_{L} & \text { if } \rho_{R} \leq \frac{1}{2} \leq \rho_{L}, \\ 1+2 \rho_{R} & \text { if } \rho_{L} \leq \frac{1}{2} \leq \rho_{R}, \\ 2\left(\rho_{L}+\rho_{R}\right) & \text { if } \frac{1}{2} \leq \rho_{L}, \rho_{R},\end{cases}
$$

which coincides with the optimal evacuation time $T_{o}$ in the region $\rho_{L}, \rho_{R}>$ $1 / 2$. We observe that $T_{\text {exit }}^{c_{o}} \in \mathbf{C}^{\mathbf{0}}\left([0,1]^{2}\right)$ and $T_{\text {exit }}^{c_{o}}\left([0,1]^{2}\right)=[1,4]$, see Figure 11. In particular, long but easy computations allow to check that Riemanntype solutions corresponding to the $\operatorname{cost} c_{o}$ are indeed continuous at $x=\xi(t)$, for all $t>0$, and the whole construction is then admissible (see figures 12 , 13 below).
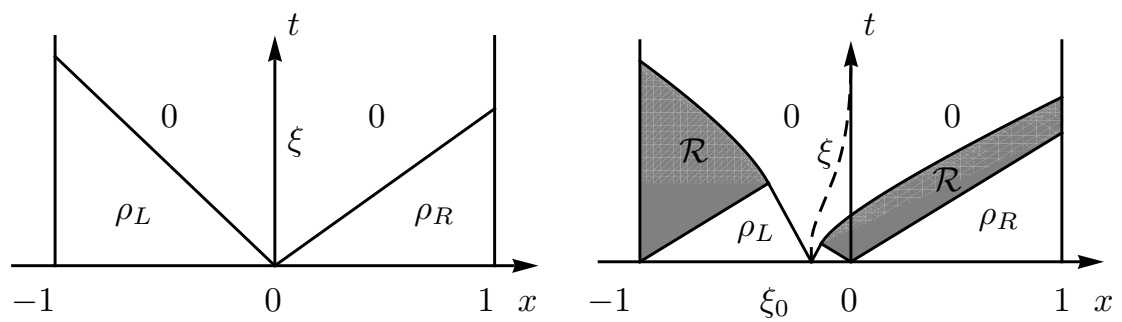

Figure 12. Solutions of the Riemann problem (1.2), (2.1), (3.1) related to the cost function $c_{o}$ given by (4.6) and corresponding to the case $\rho_{R}<\rho_{L} \leq 1 / 2$, left, and to the case $\rho_{R} \leq 1 / 2<\rho_{L}$, right. Above, the shaded areas represent rarefactions, the solid lines the shocks and the dashed lines $x=\xi(t)$. Observe that $\xi \equiv 0$ if $\rho_{R}<\rho_{L} \leq 1 / 2$.

\section{Numerical simulations}

\subsection{Solution algorithm}

5.1.1. A first-order scheme. In this section, we describe the algorithm used for numerical simulations of the model (1.1). Given an initial datum $\rho(0, x)=$ $\rho_{0}(x)$ and homogeneous Dirichlet boundary conditions (1.2), we solve (1.1) in an iterative manner at each time step, i.e.

1. Given $\rho$, solve the eikonal equation (1.1b) by the fast sweeping method, see Section 5.1.2. 

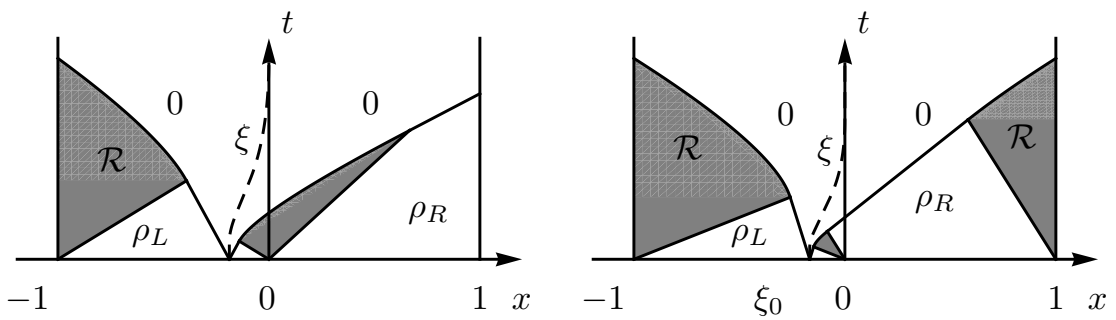

Figure 13. Solutions of the Riemann problem (1.2), (2.1), (3.1) related to the cost function $c_{o}$ given by (4.6) and corresponding to the case $\rho_{R} \leq 1 / 2<\rho_{L}$, left, and to the case $1 / 2<\rho_{R}<\rho_{L}$, right. Above, the shaded areas represent rarefactions, the solid lines the shocks and the dashed lines $x=\xi(t)$.

2. Given $\phi$, solve the non-linear conservation law (1.1a) using Godunov or Lax-Friedrichs scheme.

The domain $[-1,1]$ is divided into cells $I_{j}=\left[x_{j-1 / 2}, x_{j+1 / 2}\right]$ with centers at points $x_{j}=j \Delta x$. The explicit algorithm used to generate the approximations $\rho_{j}^{n}$ was introduced by Towers in [17] and is written in conservation form

$$
\rho_{j}^{n+1}=\rho_{j}^{n}-\frac{\Delta t}{\Delta x}\left(k_{j+\frac{1}{2}} \mathrm{~h}_{j+\frac{1}{2}}^{n}-k_{j-\frac{1}{2}} \mathrm{~h}_{j-\frac{1}{2}}^{n}\right),
$$

where $\Delta t$ is chosen to satisfy the usual CLF condition

$$
\Delta t<\frac{\Delta x}{2 \max _{j}\left|f^{\prime}\left(\rho_{j}\right)\right|} .
$$

Here $k_{i \pm 1 / 2}=\operatorname{sgn}\left(\phi_{x}\left(x_{i \pm 1 / 2}\right)\right)$. The numerical flux $\mathrm{h}_{i+1 / 2}^{n}=h\left(u^{n}, v^{n}\right)$ is chosen to be monotone and consistent, i.e. $h(\rho, \rho)=f(\rho)=\rho v(\rho)$. In order to maintain the monotonicity of the scheme, we transpose the arguments when $k_{i+1 / 2}$ changes sign, i.e.

$$
\mathrm{h}_{j+\frac{1}{2}}= \begin{cases}h\left(\rho_{i+\frac{1}{2}}, \rho_{i}\right) & \text { if } k_{i+\frac{1}{2}} \geq 0, \\ h\left(\rho_{i}, \rho_{i+\frac{1}{2}}\right) & \text { if } k_{i+\frac{1}{2}}<0 .\end{cases}
$$

Comparing the simulations below with the expected (exact) solutions (as described in Section 3.2), we see that classical finite volume schemes for conservation laws with discontinuous flux fail to capture correctly the lower trace values at the switch line $x=\xi(t)$, where the solution is discontinuous, producing non-physical oscillations. This is due to the fact that schemes of the form (5.1) force the trace values to zero, and therefore can capture correctly only continuous solutions (whose value at $x=\xi(t)$ is $\rho=0$ ). Therefore, numerical methods for (1.1) need to be further investigated in order to manage possible presence of non-classical shocks at $x=\xi(t)$. 


\subsubsection{The Fast Sweeping algorithm [19].}

Discretization. We use the following Godunov upwind difference scheme to discretize the partial differential equation at interior points:

$$
\left[\left(\phi_{i}^{h}-\phi_{x \min }^{h}\right)^{+}\right]^{2}=c\left(\rho_{i}\right)^{2} h^{2}, \quad i=2, \ldots, I-1,
$$

where $\phi_{x \min }^{h}=\min \left(\phi_{i-1}^{h}, \phi_{i+1}^{h}\right)$ and

$$
(x)^{+}= \begin{cases}x, & x>0 \\ 0, & x \leq 0 .\end{cases}
$$

Initialization. We assign exact values at grid points in or near $\{-1,1\}$ to enforce boundary condition $\phi( \pm 1)=0$. We assign large positive values at all other grid points. These points will be updated later.

Iterations. At each grid point $x_{i}$ whose value is not fixed during the initialization, compute the solution denoted by $\bar{\phi}$, of (5.3) from the current values of its neighbors $\phi_{i \pm 1}^{h}$ and then update $\phi_{i}^{h}$ to be the smaller one between $\bar{\phi}$ and $\phi_{i}^{h}$; i.e. $\phi_{i}^{\text {new }}=\min \left(\phi_{i}^{\text {old }}, \bar{\phi}\right)$. We sweep the whole domain with two alternating orderings repeatedly:

$$
\text { (1) } i=1: I, \quad(2) i=I: 1 .
$$

5.1.3. Godunov scheme. The Godunov scheme is obtained by using the exact solutions for a conservation law of type $\rho_{t}+F(\rho)_{x}=0$ with piecewise constant initial data. The numerical flux is $h(u, v)=F\left(u^{G}(v, u)\right)$, where $u^{G}(v, u)$ is the solution of the Riemann problem with left and right state $u$ and $v$ evaluated at $x=0$, where a jump on the initial data occurs.

The Godunov flux is given by

$$
h(u, v)= \begin{cases}\min _{[u, v]} F(w) & \text { if } u \leq v, \\ \max _{[u, v]} F(w) & \text { if } u \geq v .\end{cases}
$$

5.1.4. Lax-Friedrichs scheme. In [12], the authors prove convergence the of Lax-Friedrichs scheme for conservation laws with discontinuous space-time dependent flux. These results can be used in the second step to solve the conservation law. The Lax-Friedrichs flux is given by

$$
h(u, v)=\frac{1}{2}(f(u)+f(v))+\frac{\Delta x}{2 \Delta t}(u-v),
$$

and is known to display strong dissipation and dispersion.

\subsection{Solutions discontinuous at $x=\xi(t)$}

In this section, we investigate numerically the behavior of solutions that may experience a discontinuity along $x=\xi(t)$, by showing simulations with different initial conditions. The tests have been performed taking $\rho_{R}=0$ and $\rho_{L}=0.7,0.8,0.9$, and $c(\rho)=1 / v(\rho)$ as cost function. Figures 14, 15] and 16 show the results obtained with Godunov scheme on the left and the LaxFriedrichs scheme on the right, with a space step $\Delta x=0.004$. Both schemes confirm the presence of mass crossing the switch line $x=\xi(t)$ for values of $\rho_{L}$ greater than a critical value close to 0.8 , as predicted by the analysis 

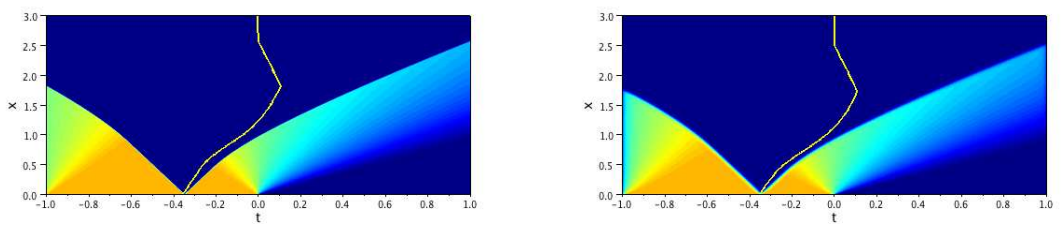

Figure 14. Case: $\rho_{L}=0.7$ and $\rho_{R}=0$
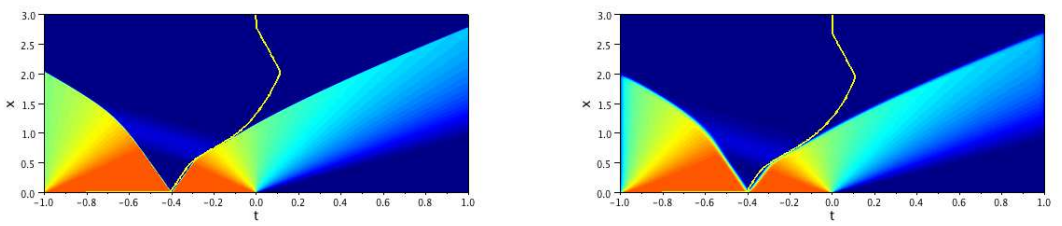

Figure 15. Case: $\rho_{L}=0.8$ and $\rho_{R}=0$
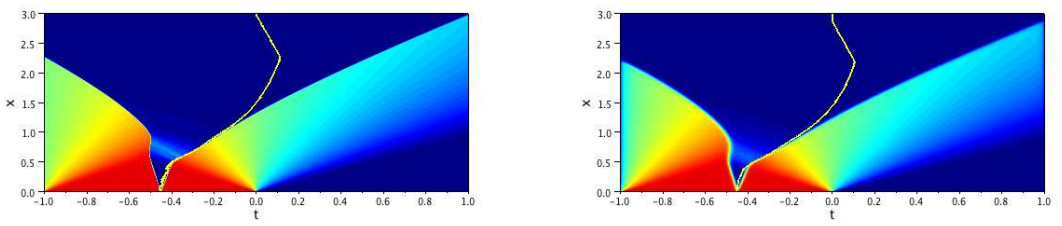

Figure 16. Case: $\rho_{L}=0.9$ and $\rho_{R}=0$

performed in Section 3. We observe that Godunov scheme presents wider oscillations around the switch line, not being able to properly capture the lower trace value. On the other side, Lax-Friedrichs schemes does not capture correctly the position of the line $x=\xi(t)$ when it is not in the vacuum region.

\subsection{Cost functions and exit times}

The following numerical tests show how the structure of the solution and the exit time vary with the choice of the cost function. We used Godunov scheme with $\Delta x=0.004$. All the tests have been performed with the initial condition given by

$$
\rho_{0}= \begin{cases}0.8 & \text { if }-0.8 \leq x \leq-0.5 \\ 0.6 & \text { if }-0.3 \leq x \leq 0.3 \\ 0.9 & \text { if } 0.4 \leq x \leq 0.75 \\ 0 & \text { elsewhere }\end{cases}
$$

as in [9, Figure 6]. In figures 17, 18 and 19 below we have depicted in yellow the corresponding trajectories $x=\xi(t)$. As expected, the choice of $c(\rho) \equiv 1$ maximizes the exit time and could be used to describe the case of a panicking 


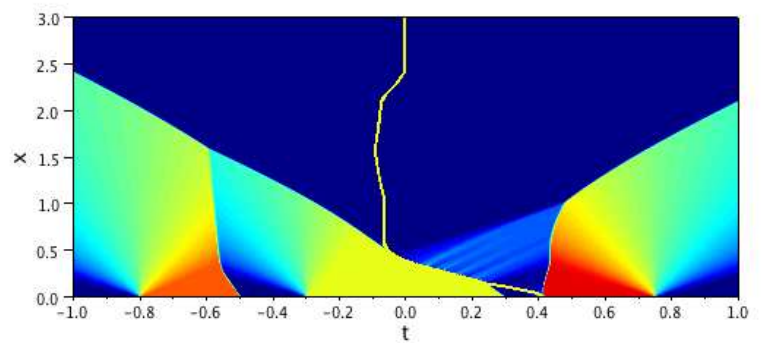

Figure 17. Case $c(\rho)=1 / v(\rho)$ : We can observe positive densities appearing on the right of $x=\xi(t)$, representing people changing advise and inverting their route. The numerically computed exit time is $T_{\text {exit }}=2.542$.

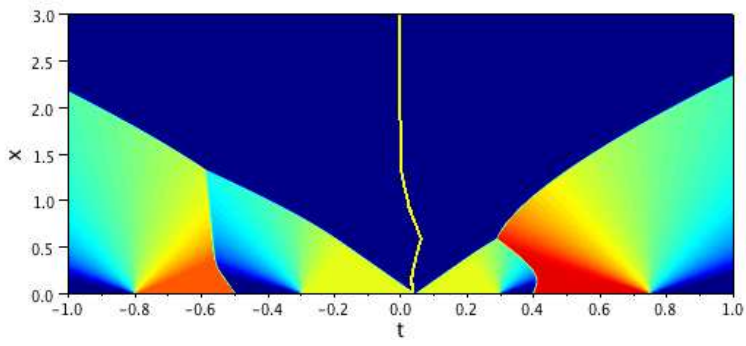

Figure 18. Case $c(\rho)=c_{o}(\rho)$ defined by (4.6): Oscillations have disappeared and $\rho(t, \xi(t) \pm)=0$. The numerically computed exit time has improved to $T_{\text {exit }}=2.474$.

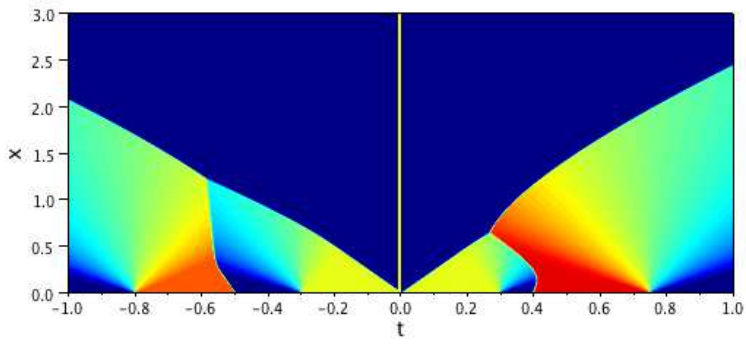

Figure 19. Case $c(\rho)=1$ : This choice should correspond to the case of a panicking crowd: people are moving towards the closer exit regardless of the presence of high densities in front of them. The numerically computed exit time has increased to $T_{\text {exit }}=2.572$. 
crowd, where people are moving towards the closer exit regardless of the density distribution. On the other hand, the choice $c(\rho)=c_{o}(\rho)$ defined by (4.6) improves (i.e., diminishes) the exit time. We remark that oscillations develop in the case of $c(\rho)=1 / v(\rho)$, thus the solution is not everywhere continuous at $x=\xi(t)$. In particular, comparing Figure 17 with [9, Figure 6], we see that oscillations appearing in first order approximations of the nonviscous conservation equation are not present in the viscous solution, which exhibits a regular profile.

\section{The eikonal equation}

In this section we give further details on equation (1.1b), for a given $\rho \in$ $\mathbf{C}^{\mathbf{0}}\left(\mathbb{R}^{+} ; \mathrm{BV}(\Omega) \cap \mathbf{L}^{\mathbf{1}}(\Omega)\right)$.

As usual in the theory of Hamilton-Jacobi equations, (1.1b) is related to the trajectories $y(t ; \alpha, x)$ of the following control system

$$
\begin{cases}y^{\prime}(t)=\alpha(t), & t>0, \\ y(0)=x, & x \in \Omega,\end{cases}
$$

where the control $\alpha=\alpha(t)$ is any measurable function $\alpha: \mathbb{R}^{+} \rightarrow[-1,1]$. Let us consider the exit-time $t(\alpha, x)$ of the trajectories of system (6.1) from $\Omega$, i.e.

$$
t(\alpha, x)=\inf \{t \geq 0: y(t ; \alpha, x) \in(\mathbb{R} \backslash \Omega)\} .
$$

For $\tau>0$ fixed, the value function

$$
V(x)=\inf _{\alpha \in A} \int_{0}^{t(\alpha, x)} g(y(s ; \alpha(s), x)) d s,
$$

where $A$ stands for the set of all measurable functions $\alpha: \mathbb{R}^{+} \rightarrow[-1,1]$, is the (unique) viscosity solution of the Hamilton-Jacobi equation

$$
H\left(x, u^{\prime}(x)\right)=g(x), \quad x \in \Omega,
$$

where the Hamiltonian $H$ is defined by

$$
H(x, p)=\sup _{a \in[-1,1]}\{-a p\}=|p| .
$$

Setting $g(x)=c(\rho(t, x))$ for $t \geq 0$ fixed, we recover 1.1b). For general reference on viscosity solutions in optimal control we refer to [2. We remark here that classical theory of viscosity solutions assumes that $g$ is continuous. The case of $g$ discontinuous, which is of interest for applications to problem (1.1), has been addressed for example in [16] (see also references therein). It is proved in particular that, if $g$ is lower semicontinuous, strictly positive and bounded, then the value function (6.2) is the unique viscosity solution of (6.3). Since $\rho(t, \cdot)$ has bounded variation, we can always consider the lower semicontinous limit at the jump points.

Finally, it is easy to see that in our case an optimal control is given by $\alpha(t ; x) \equiv \operatorname{sgn}(x)$ and, performing the change of variable $y=y(s ; \alpha(s), x)$ in (6.2) we recover (1.5). 
We can now give the following regularity properties of the function $\xi=\xi(t)$ defined by (1.6).

Lemma 6.1. Let $\rho \in \mathbf{C}^{\mathbf{0}}\left(\mathbb{R}^{+} ; B V(\Omega) \cap \mathbf{L}^{\mathbf{1}}(\Omega)\right)$. Then the corresponding function $\xi=\xi(t)$ defined by (1.6) is continuous.

Proof. From (1.6) we deduce

$$
\begin{aligned}
\int_{\xi(t)}^{\xi(s)} c(\rho(t, y))+c(\rho(s, y)) d y= & \int_{-1}^{\xi(t)} c(\rho(t, y))-c(\rho(s, y)) d y \\
& +\int_{\xi(s)}^{1} c(\rho(s, y))-c(\rho(t, y)) d y
\end{aligned}
$$

Assuming without loss of generality $\xi(t) \leq \xi(s)$ and since $c(\rho) \geq 1$, from the above equality we get

$$
\begin{aligned}
2(\xi(s)-\xi(t)) \leq & \int_{-1}^{\xi(t)}|c(\rho(t, y))-c(\rho(s, y))| d y \\
& +\int_{\xi(s)}^{1}|c(\rho(s, y))-c(\rho(t, y))| d y \\
\leq & \|c(\rho(s, \cdot))-c(\rho(t, \cdot))\|_{\mathbf{L}^{1}(\Omega)},
\end{aligned}
$$

and we conclude by the $\mathbf{L}^{\mathbf{1}}$-continuity of the function $c \circ \rho$.

Lemma 6.2. Let $\rho^{1}, \rho^{2} \in \mathbf{C}^{\mathbf{0}}\left(\mathbb{R}^{+} ; B V(\Omega) \cap \mathbf{L}^{\mathbf{1}}(\Omega)\right)$. Then for every $T>0$ the corresponding functions $\xi_{1}, \xi_{2}$ defined by (1.6) satisfy

$$
\left\|\xi_{1}-\xi_{2}\right\|_{\mathbf{L}^{\mathbf{1}}([0, T])} \leq \frac{1}{2} \mathcal{L} i p(c ;[0,1])\left\|\rho^{1}-\rho^{2}\right\|_{\mathbf{L}^{\mathbf{1}}([0, T] \times \Omega)},
$$

where $\mathcal{L}$ ip $(c ;[0,1])$ is the Lipschitz constant of $c$ on the interval $[0,1]$.

Proof. As before, from (1.6) we deduce for $t \in[0, T]$

$$
\begin{aligned}
\int_{\xi_{1}(t)}^{\xi_{2}(t)} c\left(\rho^{1}(t, y)\right)+c\left(\rho^{2}(t, y)\right) d y= & \int_{-1}^{\xi_{1}(t)} c\left(\rho^{1}(t, y)\right)-c\left(\rho^{2}(t, y)\right) d y \\
& +\int_{\xi_{2}(t)}^{1} c\left(\rho^{2}(t, y)\right)-c\left(\rho^{1}(t, y)\right) d y
\end{aligned}
$$

Assuming without loss of generality $\xi_{1}(t) \leq \xi_{2}(t)$ and since $c(\rho) \geq 1$, from the above equality we get

$$
\begin{aligned}
2\left(\xi_{2}(t)-\xi_{1}(t)\right) \leq & \int_{-1}^{\xi_{1}(t)}\left|c\left(\rho^{1}(t, y)\right)-c\left(\rho^{2}(t, y)\right)\right| d y \\
& +\int_{\xi_{2}(t)}^{1}\left|c\left(\rho^{2}(t, y)\right)-c\left(\rho^{1}(t, y)\right)\right| d y \\
\leq & \mathcal{L} i p(c ;[0,1]) \int_{-1}^{1}\left|\rho^{1}(t, y)-\rho^{2}(t, y)\right| d y
\end{aligned}
$$

Integrating the above inequality in time over the interval $[0, T]$, we get the conclusion. 


\section{References}

[1] D. Amadori and M. Di Francesco. The one-dimensional Hughes model for pedestrian flow: Riemann-type solutions. Preprint, 2011.

[2] M. Bardi and I. Capuzzo-Dolcetta. Optimal control and viscosity solutions of Hamilton-Jacobi-Bellman equations. Systems \& Control: Foundations \& Applications. Birkhäuser Boston Inc., Boston, MA, 1997. With appendices by Maurizio Falcone and Pierpaolo Soravia.

[3] C. Bardos, A. Y. le Roux, and J.-C. Nédélec. First order quasilinear equations with boundary conditions. Comm. Partial Differential Equations, 4(9):10171034, 1979.

[4] C. Chalons. Numerical approximation of a macroscopic model of pedestrian flows. SIAM J. Sci. Comput., 29(2):539-555 (electronic), 2007.

[5] R. M. Colombo, P. Goatin, and M. D. Rosini. A macroscopic model for pedestrian flows in panic situations. In Current advances in nonlinear analysis and related topics, volume 32 of GAKUTO Internat. Ser. Math. Sci. Appl., pages 255-272. Gakkōtosho, Tokyo, 2010.

[6] R. M. Colombo and M. D. Rosini. Pedestrian flows and non-classical shocks. Math. Methods Appl. Sci., 28(13):1553-1567, 2005.

[7] R. M. Colombo and M. D. Rosini. Existence of nonclassical solutions in a pedestrian flow model. Nonlinear Anal. Real World Appl., 10(5):2716-2728, 2009.

[8] C. M. Dafermos. Hyperbolic conservation laws in continuum physics, volume 325 of Grundlehren der Mathematischen Wissenschaften [Fundamental Principles of Mathematical Sciences]. Springer-Verlag, Berlin, second edition, 2005.

[9] M. Di Francesco, P. A. Markowich, J.-F. Pietschmann, and M.-T. Wolfram. On the Hughes' model for pedestrian flow: the one-dimensional case. J. Differential Equations, 250(3):1334-1362, 2011.

[10] R. L. Hughes. A continuum theory for the flow of pedestrians. Transpn. Res. $-B$, 36(6):507-535, 2002.

[11] R. L. Hughes. The flow of human crowds. In Annual review of fluid mechanics, Vol. 35, volume 35 of Annu. Rev. Fluid Mech., pages 169-182. Annual Reviews, Palo Alto, CA, 2003.

[12] K. H. Karlsen and J. D. Towers. Convergence of the Lax-Friedrichs scheme and stability for conservation laws with a discontinous space-time dependent flux. Chinese Ann. Math. Ser. B, 25(3):287-318, 2004.

[13] S. N. Kružhkov. First order quasilinear equations with several independent variables. Mat. Sb. (N.S.), 81 (123):228-255, 1970.

[14] S. Mishra. Convergence of upwind finite difference schemes for a scalar conservation law with indefinite discontinuities in the flux function. SIAM J. Numer. Anal., 43(2):559-577 (electronic), 2005.

[15] M. D. Rosini. Nonclassical interactions portrait in a macroscopic pedestrian flow model. J. Differential Equations, 246(1):408-427, 2009.

[16] P. Soravia. Boundary value problems for Hamilton-Jacobi equations with discontinuous Lagrangian. Indiana Univ. Math. J., 51(2):451-477, 2002.

[17] J. D. Towers. Convergence of a difference scheme for conservation laws with a discontinuous flux. J. Numer. Anal., 38(2):681-698, 2000. 
[18] A. Vasseur. Strong traces for solutions of multidimensional scalar conservation laws. Arch. Ration. Mech. Anal., 160(3):181-193, 2001.

[19] H. Zhao. A fast sweeping method for eikonal equations. Math. Comp., 74(250):603-627 (electronic), 2005.

Nader El-Khatib

Computer Science and Mathematics Department

Lebanese American University

Byblos Campus - P.O. Box 36

Byblos

Lebanon

e-mail: nader.elkhatib@lau.edu.lb

Paola Goatin

INRIA Sophia Antipolis - Méditerranée

EPI OPALE

2004, route des Lucioles - BP 93

06902 Sophia Antipolis Cedex

France

e-mail: paola.goatin@inria.fr

Massimiliano D. Rosini

ICM, University of Warsaw

ul. Pawinskiego 5a

02-106 Warszawa

Poland

e-mail: mrosini@icm.edu.pl 